\title{
Velocity Evolution and the Intrinsic Color of Type la Supernovae
}

\section{Citation}

Foley, Ryan J., Nathan E. Sanders, and Robert P. Kirshner. 2011. "VELOCITY EVOLUTION AND THE INTRINSIC COLOR OF TYPE la SUPERNOVAE." The Astrophysical Journal 742 (2): 89. https://doi.org/10.1088/0004-637x/742/2/89.

\section{Permanent link}

http://nrs.harvard.edu/urn-3:HUL.InstRepos:41399778

\section{Terms of Use}

This article was downloaded from Harvard University's DASH repository, and is made available under the terms and conditions applicable to Other Posted Material, as set forth at http:// nrs.harvard.edu/urn-3:HUL.InstRepos:dash.current.terms-of-use\#LAA

\section{Share Your Story}

The Harvard community has made this article openly available.

Please share how this access benefits you. Submit a story.

Accessibility 


\title{
VELOCITY EVOLUTION AND THE INTRINSIC COLOR OF TYPE Ia SUPERNOVAE
}

\author{
Ryan J. Foley ${ }^{1}$, Nathan E. SANDERs, and Robert P. Kirshner \\ Harvard-Smithsonian Center for Astrophysics, 60 Garden Street, Cambridge, MA 02138, USA; rfoley@ cfa.harvard.edu \\ Received 2011 July 18; accepted 2011 September 11; published 2011 November 9
}

\begin{abstract}
To understand how best to use observations of Type Ia supernovae (SNe Ia) to obtain precise and accurate distances, we investigate the relations between spectra of SNe Ia and their intrinsic colors. Using a sample of 1630 optical spectra of $255 \mathrm{SNe}$, based primarily on data from the CfA Supernova Program, we examine how the velocity evolution and line strengths of Si II $\lambda 6355$ and Ca II $\mathrm{H} \& \mathrm{~K}$ are related to the $B-V$ color at peak brightness. We find that the maximum-light velocity of Si II $\lambda 6355$ and $\mathrm{Ca}$ II $\mathrm{H} \& \mathrm{~K}$ and the maximum-light pseudo-equivalent width of Si II $\lambda 6355$ are correlated with intrinsic color, with intrinsic color having a linear relation with the Si II $\lambda 6355$ measurements. Ca II H\&K does not have a linear relation with intrinsic color, but lower-velocity $\mathrm{SNe}$ tend to be intrinsically bluer. Combining the spectroscopic measurements does not improve intrinsic color inference. The intrinsic color scatter is larger for higher-velocity SNe Ia-even after removing a linear trend with velocity -indicating that lower-velocity SNe Ia are more "standard crayons." Employing information derived from SN Ia spectra has the potential to improve the measurements of extragalactic distances and the cosmological properties inferred from them.
\end{abstract}

Key words: distance scale - dust, extinction - supernovae: general

Online-only material: color figures, machine-readable tables

\section{INTRODUCTION}

Type Ia supernovae (SNe Ia) are very good distance indicators after making an empirical correction based on their light-curve shape and color (Phillips 1993; Riess et al. 1996). Using these relations, large SN Ia samples have a precision of $\sim 8 \%$ for distance estimates (e.g., Hicken et al. 2009a). This level of precision is adequate to determine that the expansion of the universe is accelerating (Riess et al. 1998; Perlmutter et al. 1999) and constrain the equation-of-state parameter of dark energy (Wood-Vasey et al. 2007; Riess et al. 2007; Hicken et al. 2009b; Kessler et al. 2009; Amanullah et al. 2010; Conley et al. 2011; Sullivan et al. 2011). SNe Ia are even better distance indicators in the rest-frame near-infrared (NIR; Mandel et al. 2009, 2011); however, low-redshift NIR samples are small and high-redshift NIR samples do not yet exist.

Decades ago, Branch (1987) showed that not all SNe Ia have the same ejecta velocity, as probed by the blueshift of spectral features. Branch et al. (1988) noted that a sample of $\mathrm{SNe}$ Ia had a very broad distribution of Si II $\lambda 6355$ velocity near maximum brightness and concluded: "SNe Ia are not observationally homogeneous. The only way to maintain that they are physically homogeneous would be to postulate that they are identical but asymmetrical." Benetti et al. (2005) showed that the velocity gradient for the Si II $\lambda 6355$ feature did not correlate with light-curve shape for spectroscopically normal $\mathrm{SNe}$ Ia. This observation provided further proof that $\mathrm{SNe}$ Ia were heterogeneous, but also showed that some properties of a SN Ia did not depend on the width of its light curve.

Several studies have shown that SN Ia spectral properties are related to their photometric properties (e.g., Nugent et al. 1995). Some studies examined the correlation between Hubble residuals after light-curve shape correction and spectroscopic properties to produce a smaller Hubble scatter (Foley et al. 2008b; Bailey et al. 2009; Blondin et al. 2011b; Chotard

\footnotetext{
1 Clay Fellow.
}

et al. 2011; Nordin et al. 2011b). However, using this simple approach with only optical spectra provides only minor gains over photometry alone (Blondin et al. 2011b).

In a previous study, Foley \& Kasen (2011, hereafter FK11) showed that the maximum-light intrinsic color of $\mathrm{SNe}$ Ia is highly correlated with their ejecta velocity as probed by the Si II $\lambda 6355$ feature, $v_{\mathrm{Si} \text { II }}$. Accounting for this correlation both substantially improves the precision and reduces potential biases of SN Ia distance measurements. Theoretically, this is understood as increased line blanketing in the higher-velocity $\mathrm{SNe}$, depressing the $B$-band flux relative to the $V$-band flux and causing a redder $B-V$ color. Another theoretical expectation is that higher-velocity SNe Ia have more absorption from lower excitation (e.g., Fe II versus Fe III) lines, which are distributed such that higher-velocity SNe Ia should have more absorption in the near-ultraviolet. Nonetheless, not all SN Ia models show the faster-redder relation found in the data (Blondin et al. 2011a).

Although not necessarily the cause of the relation between intrinsic color and $v_{\mathrm{Si} \text { II }}$, the observations are naturally explained by asymmetric explosions (FK11), as originally suggested by Branch et al. (1988). An asymmetric explosion model was invoked to explain a striking relation between the velocity gradient of the Si II $\lambda 6355$ feature, $\dot{v}_{\mathrm{Si} \text { II }}$, and the velocity offset of nebular lines at late times (Maeda et al. 2010). Leonard et al. (2005) found that SNe Ia with high-velocity features had stronger Si II $\lambda 6355$ line polarization than those without the features. Maund et al. (2010) expanded upon this work and found a correlation between the amount of polarization in the Si II feature and $\dot{v}_{\mathrm{Si} \text { iI }}$, suggesting that $\dot{v}_{\mathrm{Si} \text { iI }}$ is an excellent probe of asymmetry of the outer layers of the SN ejecta. Since $v_{\mathrm{Si} \text { II }}$ is an excellent proxy for $\dot{v}_{\mathrm{SiII}}$ (Wang et al. 2009a, hereafter W09), one expects $v_{\mathrm{Si} \text { II }}, \dot{v}_{\mathrm{Si} \text { II }}$, intrinsic color at maximum brightness, nebular line velocity offsets, and $\mathrm{Si}$ II polarization should all be highly correlated. Maeda et al. (2011) confirmed one of these assumptions, showing that nebular line velocity offsets are correlated with maximum-light color. Of all of these observables, $v_{\mathrm{Si} \text { II }}$ is the easiest to obtain at low redshift (and is 
always obtained if $\dot{v}_{\mathrm{Si} \text { II }}$ or polarization measurements are made) and is the only observable known to correlate with intrinsic color that we can realistically obtain for high- $z$ SNe.

Besides line velocity, which is determined by the minimum of an absorption feature (corresponding to its maximum absorption), one might expect that the total amount of absorption, as measured by either the FWHM or pseudo-equivalent width (pEW) of a feature, to strongly correlate with intrinsic color. Both the FWHM and pEW are alternative kinematic probes, measuring the span of the absorbing material in velocity space and may have more physical motivation for a correlation with intrinsic color than absorption velocity.

Although Si II $\lambda 6355$ is a clean, isolated, strong feature in SN Ia spectra, there is no guarantee that its properties are the most informative for determining intrinsic color. Furthermore, Si II $\lambda 6355$ redshifts out of the optical window at $z \approx 0.4$. Therefore, any relation determined with Si II $\lambda 6355$ cannot be applied to most high- $z$ SNe Ia. Another strong feature in SN Ia spectra that has a large velocity distribution and strong velocity evolution with time is Ca II H\&K. FK11 suggested that this feature, which is much bluer than Si II $\lambda 6355$ and can be observed in the optical window up to $z \approx 1.2$, may be an alternative way to determine intrinsic color. FK11 found that the model spectra of Kasen \& Plewa (2007) show a strong relation between intrinsic color and $v_{\mathrm{CaH} \& \mathrm{~K}}$, similar to what was found with $v_{\mathrm{Si} \text { II }}$.

Current and previous high- $z$ SN Ia samples typically have a single near maximum brightness observer-frame optical spectrum per SN (e.g., Zheng et al. 2008; Foley et al. 2009; Walker et al. 2011), making measurements of $\dot{v}$ impossible. Many current and future surveys intend to use photometric-only samples of $\mathrm{SNe}$ for cosmological studies where only a small subsample of SNe will have spectra. These samples will also not have the spectral series necessary to determine velocity gradients for high- $z$ SNe Ia. Moreover, if intrinsic color can be determined by a single spectrum, telescope time can be used to make this measurement for more $\mathrm{SNe}$ than one could if a time series is required.

Since FK11 used the W09 sample, which did not provide spectra or velocity measurements, but only a designation of "Normal" or "High-Velocity" for SNe, FK11 was unable to investigate exactly how intrinsic color depends on ejecta velocity. Similarly, W09 did not provide any FWHM or pEW measurements or any measurements for Ca II H\&K. In this paper, we use the large and homogeneous CfA sample of SN Ia spectra (S. Blondin et al. 2012, in preparation), supplemented by literature data (Section 2), to construct a homogeneous sample which reduces potential systematic differences, determine a relation between $\dot{v}$ and $v(t)$ for the two strongest features in a maximum-light SN Ia spectrum, Si II $\lambda 6355$ and Ca II H\&K (Section 3), use the same data to determine a relation between pEW for these features and their gradients (Section 4), use those relations to measure maximum-light values for a large sample of $\mathrm{SNe}$, allowing a direct comparison of these $\mathrm{SNe}$ (Sections 3 and 4 ), and reexamine the relation between ejecta kinematics-as determined by both the width and velocity of the minimum of the absorption for Si II $\lambda 6355$ and $\mathrm{Ca}$ II $\mathrm{H} \& \mathrm{~K}$ - and intrinsic color (Section 5). We find that the maximum-light velocity of Si II $\lambda 6355$ and $\mathrm{Ca}$ II $\mathrm{H} \& \mathrm{~K}$ and the maximum-light $\mathrm{pEW}$ of Si II $\lambda 6355$ are correlated with intrinsic color. The intrinsic color scatter is larger for higher-velocity $\mathrm{SNe} \mathrm{Ia}$ - even after removing a linear trend with velocity-indicating that lower-velocity SNe Ia are more "standard crayons." We discuss these results and conclude in Section 6.

See Peek \& Graves (2010) for a description of this nomenclature.

\section{DATA}

For our study of how SN Ia intrinsic color is related to kinematics, we require a large sample of SNe Ia with both photometry (to measure colors) and spectra (to measure kinematic probes). Below we describe the data used for our sample.

Additionally, we use values reported by W09. Since W09 do not report velocity measurements (only a designation of "Normal" or "High-Velocity"), it is not sufficient for use as a spectroscopic sample. They do report some relevant photometric measurements (specifically $M_{V}$ at peak, $\Delta m_{15}(B)$, and $\left.B_{\max }-V_{\max }\right)$. However, the photometric data come from varied sources (Hamuy et al. 1996a, 1996b; CfA1; Riess et al. 1999; all compiled by Reindl et al. 2005; CfA2; Jha et al. 2006; CfA3; Hicken et al. 2009a; and preliminary Lick Observatory Supernova Search (LOSS) light curves later published by Ganeshalingam et al. 2010), and there are potentially systematic differences between each data set. A compilation of several photometric sources can have major differences, even after placing all photometry on a standard system. At the very least, $S$ corrections are typically necessary to achieve agreement at the $0.01 \mathrm{mag}$ level (Stritzinger et al. 2002).

Although we use the data presented by W09 for an initial analysis (see Section 5), we do not combine data from W09 with other data for our final analysis.

\subsection{Photometry}

Our photometric data come from two sources: CfA3 (Hicken et al. 2009a) and LOSS (Ganeshalingam et al. 2010). Each sample is large, and there is substantial overlap in SNe between the two groups. Each group derived parameters from their light curves. Specifically, they each measure $B_{\max }, V_{\max }$, and $\Delta m_{15}(B)$. Ganeshalingam et al. (2010) used a combination of template fitting (when the data are good and similar to normal SN Ia light curves) and polynomial fitting (when template fitting fails). Hicken et al. (2009a) only used polynomial fitting. The template fitting is more sophisticated and should produce better measurements. We therefore use LOSS-derived parameters for SNe Ia that have both LOSS and CfA3 data.

For 40, 42, and $29 \mathrm{SNe} \mathrm{Ia}$ in common for $\mathrm{CfA}^{3}$ and LOSS, we find average offsets of $\left\langle B_{\max }^{\mathrm{CfA}}-B_{\max }^{\mathrm{LOSS}}\right\rangle=0.020 \pm$ $0.010 \mathrm{mag},\left\langle V_{\max }^{\mathrm{CfA}}-V_{\max }^{\mathrm{LOSS}}\right\rangle=-0.006 \pm 0.008 \mathrm{mag}$, and $\left\langle\Delta m_{15}^{\mathrm{CfA}}-\Delta m_{15}^{\mathrm{LOSS}}\right\rangle=0.008 \pm 0.017 \mathrm{mag}$, respectively. Furthermore, for $40 \mathrm{SNe}$ in common, we find $\left\langle\left(B_{\max }-V_{\max }\right)^{\mathrm{CfA}}-\right.$ $\left.\left(B_{\max }-V_{\max }\right)^{\text {LOSS }}\right\rangle=0.025 \pm 0.011 \mathrm{mag}$. Considering that FK11 found an offset in $B_{\max }-V_{\max }$ of $\sim 0.1 \mathrm{mag}$ for low- and high-velocity SNe Ia, this offset could cause enough scatter to reduce the significance of potential correlations.

Ganeshalingam et al. (2010) examined the light curves in common between the LOSS and CfA3 sample. Interpolating LOSS light curves, they examined differences between individual light-curve points. They found average offsets of $0.011 \pm 0.006(0.016 \pm 0.005)$ and $-0.006 \pm 0.004(-0.010 \pm$ $0.004)$ mag for $B$ and $V$ using only data brighter than magnitude 18 (all data), respectively. These measurements are consistent with our finding for the derived parameters. Confirmation with the authors indicates that extinction corrections and $K$ corrections were performed in the same way (M. Ganeshalingam \&

\footnotetext{
3 We correct the CfA3 SN 2008bf photometry for an incorrect calibration which affected the light curves by $0.07 \mathrm{mag}$ in $B$ and $0.13 \mathrm{mag}$ in $V$. After correction, the peak magnitudes are $B_{\max }=15.65 \pm 0.04$ mag and $V_{\max }=15.81 \pm 0.04 \mathrm{mag}$ (M. Hicken 2011, private communication).
} 
Table 1

Object Information

\begin{tabular}{|c|c|c|c|c|c|c|c|c|}
\hline SN & $z_{\mathrm{CMB}}$ & $\begin{array}{l}V_{\max }{ }^{\mathrm{a}} \\
(\mathrm{mag})\end{array}$ & $\begin{array}{l}\Delta m_{15}(B)^{\mathrm{b}} \\
(\mathrm{mag})\end{array}$ & $\begin{array}{c}B_{\max }-V_{\max } \\
(\mathrm{mag})\end{array}$ & $\begin{array}{c}v_{\mathrm{SiII}}^{0} \\
\left(10^{3} \mathrm{~km} \mathrm{~s}^{-1}\right)\end{array}$ & $\begin{array}{c}\mathrm{pEW}_{0}(\mathrm{Si} \mathrm{II}) \\
(\AA)\end{array}$ & $\begin{array}{c}v_{\mathrm{CaH} \& \mathrm{~K}^{\mathrm{c}}}^{0} \\
\left(10^{3} \mathrm{~km} \mathrm{~s}^{-1}\right)\end{array}$ & $\begin{array}{c}\mathrm{pEW}_{0}(\mathrm{Ca} \text { II }) \\
(\AA)\end{array}$ \\
\hline $1981 \mathrm{~B}^{\mathrm{d}}$ & 0.0036 & $11.90(0.05)$ & $1.08(0.03)$ & $0.01(0.06)$ & $-11.87(0.22)$ & $128.8(6.0)$ & $-15.31(0.57)$ & $112.7(6.9)$ \\
\hline $1986 \mathrm{G}$ & 0.0027 & $11.12(0.06)$ & $1.75(0.00)$ & $0.88(0.09)$ & $-10.48(0.22)$ & $\ldots$ & $\ldots$ & $\ldots$ \\
\hline 1989B & 0.0035 & $11.89(0.05)$ & $1.16(0.02)$ & $0.32(0.10)$ & $-10.56(0.22)$ & $121.1(6.0)$ & $-11.65(0.57)$ & $106.4(6.9)$ \\
\hline $1990 \mathrm{~N}^{\mathrm{d}}$ & 0.0051 & $12.66(0.04)$ & $1.11(0.01)$ & $0.01(0.06)$ & $-9.38(0.22)$ & 84.7 (6.0) & $\ldots$ & $\ldots$ \\
\hline 19900 & 0.0306 & $\ldots$ & $0.95(0.02)$ & $\ldots$ & $-11.99(0.22)$ & $\ldots$ & $\ldots$ & $\ldots$ \\
\hline $1991 \mathrm{M}$ & 0.0076 & $\ldots$ & $\ldots$ & $\ldots$ & $-12.81(0.22)$ & $\ldots$ & $\ldots$ & $\ldots$ \\
\hline $1991 \mathrm{~T}$ & 0.0069 & $11.46(0.02)$ & $0.93(0.01)$ & $0.12(0.03)$ & $-9.62(0.22)$ & $\ldots$ & $\ldots$ & $\ldots$ \\
\hline $1991 \mathrm{bg}$ & 0.0045 & $13.86(0.04)$ & $1.94(0.00)$ & $0.71(0.06)$ & $-10.04(0.22)$ & & $\ldots$ & $\ldots$ \\
\hline $1992 \mathrm{~A}$ & 0.0058 & $12.49(0.01)$ & $1.48(0.00)$ & $0.02(0.02)$ & $-14.04(0.22)$ & $109.6(6.0)$ & $-17.22(0.57)$ & $98.9(6.9)$ \\
\hline 1992ag & 0.0259 & $16.17(0.06)$ & $1.11(0.09)$ & $0.01(0.07)$ & $-11.74(0.30)$ & $\ldots$ & $\ldots$ & $\ldots$ \\
\hline 1992al & 0.0141 & $14.56(0.04)$ & $1.10(0.08)$ & $-0.13(0.04)$ & $-11.26(0.30)$ & $\ldots$ & $\ldots$ & $\ldots$ \\
\hline $1993 \mathrm{ac}$ & 0.0493 & $\ldots$ & $1.19(0.10)$ & $\ldots$ & $-13.21(0.22)$ & $144.6(6.0)$ & $-17.93(0.57)$ & $103.0(6.9)$ \\
\hline 1994D & 0.0041 & $11.83(0.02)$ & $1.42(0.00)$ & $-0.04(0.04)$ & $-11.24(0.24)$ & $100.6(6.0)$ & $-11.53(0.58)$ & $92.4(6.9)$ \\
\hline 1994M & 0.0243 & $\ldots$ & $1.35(0.03)$ & $\ldots$ & $-12.36(0.27)$ & $118.6(6.1)$ & $\ldots$ & $\ldots$ \\
\hline $1994 S$ & 0.0160 & $14.79(0.06)$ & $1.02(0.00)$ & $-0.02(0.08)$ & $-10.72(0.24)$ & $85.0(6.0)$ & $\ldots$ & $117.8(6.9)$ \\
\hline $1994 \mathrm{~T}$ & 0.0356 & $17.15(0.04)$ & $1.51(0.08)$ & $0.18(0.05)$ & $-13.43(0.30)$ & $\ldots$ & $\ldots$ & $\ldots$ \\
\hline $1994 a e^{d}$ & 0.0062 & $13.00(0.03)$ & $1.09(0.01)$ & $-0.04(0.07)$ & $-10.98(0.23)$ & $81.6(6.0)$ & $-12.67(0.59)$ & $110.0(6.8)$ \\
\hline $1995 \mathrm{D}$ & 0.0078 & $13.26(0.05)$ & $0.98(0.01)$ & $-0.10(0.07)$ & $-10.33(0.23)$ & $\ldots$ & $\ldots$ & $\ldots$ \\
\hline $1995 \mathrm{E}$ & 0.0121 & $15.98(0.05)$ & $1.18(0.01)$ & $0.70(0.07)$ & $-11.16(0.24)$ & $98.9(6.0)$ & $-15.79(1.08)$ & $103.0(7.8)$ \\
\hline $1995 \mathrm{al}^{\mathrm{d}}$ & 0.0067 & $13.20(0.05)$ & $0.93(0.03)$ & $0.13(0.07)$ & $-13.01(0.23)$ & $\ldots$ & $\ldots$ & $\ldots$ \\
\hline $1996 \mathrm{C}$ & 0.0275 & $\ldots$ & $0.97(0.01)$ & $\ldots$ & $-10.69(0.22)$ & $\ldots$ & $\ldots$ & $\ldots$ \\
\hline 1996X & 0.0078 & $13.03(0.04)$ & $1.24(0.00)$ & $-0.05(0.05)$ & $-11.17(0.22)$ & $90.1(6.0)$ & $-11.70(0.57)$ & $80.7(6.9)$ \\
\hline $1996 Z$ & 0.0085 & $\ldots$ & $1.22(0.10)$ & $\ldots$ & $-12.14(0.23)$ & $110.6(6.0)$ & $-16.53(0.57)$ & $61.6(6.9)$ \\
\hline 1996ai & 0.0037 & $\ldots$ & $0.96(0.02)$ & $\ldots$ & $-10.82(0.22)$ & $\ldots$ & $\ldots$ & $\ldots$ \\
\hline 1996bk & 0.0070 & $\ldots$ & $1.78(0.01)$ & $\ldots$ & $-12.16(0.22)$ & $\ldots$ & $\ldots$ & $\cdots$ \\
\hline 1996bl & 0.0348 & $\ldots$ & $1.10(0.08)$ & $\ldots$ & $-12.34(0.24)$ & $89.0(6.1)$ & $\ldots$ & $\ldots$ \\
\hline 1996bo & 0.0163 & $15.51(0.04)$ & $1.20(0.01)$ & $0.31(0.06)$ & $-12.25(0.24)$ & $133.5(6.1)$ & $\ldots$ & $\ldots$ \\
\hline $1997 \mathrm{E}$ & 0.0133 & $15.08(0.07)$ & $1.46(0.02)$ & $0.03(0.09)$ & $-12.01(0.23)$ & $120.6(6.0)$ & $\ldots$ & $\ldots$ \\
\hline $1997 \mathrm{Y}$ & 0.0165 & $\ldots$ & $1.17(0.02)$ & $\ldots$ & $-11.03(0.22)$ & $101.1(6.0)$ & $\ldots$ & $\ldots$ \\
\hline 1997bp & 0.0094 & $13.74(0.03)$ & $0.96(0.03)$ & $0.10(0.04)$ & $-15.62(0.22)$ & $\ldots$ & $\ldots$ & $\ldots$ \\
\hline $1997 \mathrm{bq}$ & 0.0095 & & $1.01(0.05)$ & & $-14.24(0.23)$ & $156.0(6.0)$ & $\ldots$ & $\ldots$ \\
\hline $1997 \mathrm{br}$ & 0.0081 & $13.43(0.08)$ & $1.10(0.03)$ & $0.16(0.13)$ & $-11.60(0.22)$ & $\ldots$ & $-15.53(0.57)$ & $51.8(6.9)$ \\
\hline $1997 \mathrm{cn}$ & 0.0176 & $\ldots$ & $1.90(0.05)$ & $\ldots$ & $-9.61(0.22)$ & $\ldots$ & $\ldots$ & $\ldots$ \\
\hline $1997 \mathrm{do}$ & 0.0105 & $\ldots$ & $0.94(0.04)$ & $\ldots$ & $-13.38(0.25)$ & $\ldots$ & $\ldots$ & $\ldots$ \\
\hline $1997 \mathrm{dt}$ & 0.0061 & $\ldots$ & $1.04(0.15)$ & $\ldots$ & $-11.33(0.22)$ & $82.6(6.0)$ & $\ldots$ & $\ldots$ \\
\hline
\end{tabular}

Notes. As described in the text, all photometry has been places on the LOSS system. Uncertainties are in parentheses.

${ }^{a}$ Corrected for Milky Way extinction using the reddening maps of Schlegel et al. (1998), as performed by Hicken et al. (2009a) and Ganeshalingam et al. (2010).

b When color information is available, $\Delta m_{15}(B)$ has been corrected for host-galaxy extinction using the method similar to that of Phillips et al. (1999); $\Delta m_{15}(B)=\Delta m_{15, \text { obs }}(B)+0.1 \times\left(\left(B_{\max }-V_{\max }\right)+0.081\right)$ (see Section 5.2). The values for $B_{\max }-V_{\max }$ include a correction for Milky Way extinction, so that correction is inherent in this process.

c Values given for the "red" component.

d Cepheid object with distance modulus from Riess et al. (2011). The redshifts listed for these SNe yield appropriate distance moduli using $H_{0}=70.5 \mathrm{~km} \mathrm{~s}^{-1} \mathrm{Mpc}^{-1}$.

(This table is available in its entirety in a machine-readable form in the online journal. A portion is shown here for guidance regarding its form and content.)

M. Hicken 2011, private communication), leaving only $S$ corrections as a possible difference. Since a SN spectrum changes with time, the $S$ corrections should also change with time. Therefore, making corrections to derived peak magnitudes using the offset we found above is likely more accurate than that found by Ganeshalingam et al. (2010), which used the full light curve.

Considering the relatively large offsets, we correct the CfA3 photometry to match the LOSS photometry, which is already the default photometry when there are data from both samples.

All derived values presented by Hicken et al. (2009a) and Ganeshalingam et al. (2010) are both $K$ correct and deredden for Milky Way reddening. We present the combined derived light-curve parameters in Table 1.

\subsection{Spectroscopy}

\subsubsection{CfA Spectral Sample}

Over the last two decades, the CfA Supernova Program has observed hundreds of SNe Ia, mostly with the FAST spectrograph (Fabricant et al. 1998) mounted on the $1.5 \mathrm{~m}$ telescope at the F. L. Whipple Observatory. The data have been reduced in a consistent manner (Matheson et al. 2008; Blondin et al. 2011b; S. Blondin et al. 2012, in preparation), producing well-calibrated spectra.

For SNe Ia in the sample with a measured time of maximum brightness from light curves, $v_{\mathrm{Si} \text { II }}$ and $v_{\mathrm{CaH} \& \mathrm{~K}}$ have been measured (S. Blondin et al. 2012, in preparation). Briefly, this is achieved by first generating a smoothed spectrum using an inverse-variance Gaussian filter (Blondin et al. 2006), and the 
Table 2

Spectral Properties

\begin{tabular}{|c|c|c|c|c|c|c|c|}
\hline SN & $\begin{array}{l}\text { Phase } \\
\text { (days) }\end{array}$ & $\begin{array}{c}v_{\mathrm{Si} \mathrm{II}} \\
\left(10^{3} \mathrm{~km} \mathrm{~s}^{-1}\right)\end{array}$ & $\begin{array}{c}\mathrm{pEW}(\mathrm{Si} \text { II }) \\
(\AA)\end{array}$ & $\begin{array}{c}v_{\text {Ca II }, \text { blue }} \\
\left(10^{3} \mathrm{~km} \mathrm{~s}^{-1}\right)\end{array}$ & $\begin{array}{c}v_{\text {CaII, } r e d} \\
\left(10^{3} \mathrm{~km} \mathrm{~s}^{-1}\right)\end{array}$ & $\begin{array}{c}\mathrm{pEW}(\mathrm{Ca} \text { II }) \\
(\AA)\end{array}$ & Ref. \\
\hline 1981B & -1.89 & $-12.05(0.06)$ & $128.6(0.1)$ & $\ldots$ & $-15.60(0.06)$ & $115.8(0.1)$ & 1 \\
\hline 1981B & 15.00 & $-10.83(0.06)$ & $218.2(0.1)$ & $\ldots$ & $-13.27(0.06)$ & $79.6(0.1)$ & 1 \\
\hline 1981B & 17.98 & $-10.39(0.06)$ & $169.4(0.1)$ & $\ldots$ & $-13.10(0.06)$ & $74.4(0.1)$ & 1 \\
\hline 1981B & 21.96 & $-10.00(0.06)$ & $252.0(0.1)$ & $\ldots$ & & $78.7(0.1)$ & 1 \\
\hline $1986 \mathrm{G}$ & -4.82 & $-11.38(0.05)$ & $140.1(0.1)$ & $\ldots$ & $-16.26(0.05)$ & $100.1(0.1)$ & 1 \\
\hline $1986 \mathrm{G}$ & -3.82 & $-11.55(0.05)$ & $131.0(0.1)$ & $\ldots$ & $-15.89(0.05)$ & $110.1(0.1)$ & 1 \\
\hline $1986 \mathrm{G}$ & -3.12 & $-11.30(0.05)$ & $134.0(0.1)$ & $\ldots$ & $\ldots$ & . & 2 \\
\hline $1986 \mathrm{G}$ & -2.82 & $-11.15(0.05)$ & $131.2(0.1)$ & $\ldots$ & $-16.68(0.05)$ & $109.2(0.1)$ & 1 \\
\hline $1986 \mathrm{G}$ & -2.32 & $-11.20(0.05)$ & $126.4(0.1)$ & $\ldots$ & $-17.17(0.05)$ & $92.3(0.1)$ & 2 \\
\hline 1986G & -1.33 & $-10.91(0.05)$ & $124.8(0.1)$ & $\ldots$ & $-17.32(0.05)$ & $90.6(0.1)$ & 2 \\
\hline $1986 \mathrm{G}$ & -0.83 & $-10.86(0.05)$ & $133.2(0.1)$ & $\ldots$ & $\ldots$ & $\ldots$ & 1 \\
\hline $1986 \mathrm{G}$ & -0.33 & $-10.13(0.05)$ & $122.7(0.1)$ & $\ldots$ & $-16.45(0.05)$ & $97.1(0.1)$ & 2 \\
\hline $1986 \mathrm{G}$ & 0.17 & $-10.47(0.05)$ & $137.2(0.1)$ & $\ldots$ & $-15.26(0.05)$ & $115.0(0.1)$ & 1 \\
\hline $1986 \mathrm{G}$ & 0.67 & $-10.51(0.05)$ & $117.7(0.1)$ & $\ldots$ & $-14.77(0.05)$ & $103.7(0.1)$ & 2 \\
\hline $1986 \mathrm{G}$ & 1.17 & $-10.47(0.05)$ & $131.2(0.1)$ & $\ldots$ & $-15.10(0.05)$ & $111.7(0.1)$ & 1 \\
\hline 1989B & -7.21 & $-11.40(0.05)$ & $121.7(0.1)$ & $-17.88(0.05)$ & $-12.48(0.05)$ & $126.1(0.1)$ & 1 \\
\hline 1989B & -1.37 & $-10.64(0.05)$ & $120.6(0.1)$ & $-16.85(0.05)$ & $-11.60(0.05)$ & $108.5(0.1)$ & 1 \\
\hline 1989B & 2.60 & $-10.65(0.05)$ & $119.4(0.1)$ & $-15.09(0.05)$ & $-11.93(0.50)$ & $129.1(0.1)$ & 1 \\
\hline 1989B & 4.56 & $-10.06(0.05)$ & $131.6(0.1)$ & $-15.45(0.05)$ & $-11.92(0.50)$ & $99.7(0.1)$ & 1 \\
\hline 1989B & 6.57 & $-10.17(0.05)$ & $130.8(0.1)$ & $-15.48(0.05)$ & $-12.11(0.50)$ & $99.1(0.1)$ & 1 \\
\hline 1989B & 8.57 & $-10.04(0.05)$ & $136.1(0.1)$ & $-14.49(0.05)$ & $-11.72(0.50)$ & $95.4(0.1)$ & 1 \\
\hline 1989B & 10.56 & $-10.04(0.05)$ & $144.5(0.1)$ & $-15.29(0.05)$ & $-12.31(0.05)$ & $84.7(0.1)$ & 1 \\
\hline 1989B & 11.54 & $-9.56(0.05)$ & $156.1(0.1)$ & $-15.09(0.05)$ & $-11.92(0.05)$ & $79.4(0.1)$ & 1 \\
\hline 1989B & 12.52 & $-9.56(0.05)$ & $130.6(0.1)$ & $-15.09(0.05)$ & $-11.14(0.05)$ & $68.8(0.1)$ & 1 \\
\hline 1989B & 13.53 & $-9.43(0.05)$ & $156.0(0.1)$ & $\ldots$ & $\ldots$ & $\ldots$ & 1 \\
\hline 1989B & 15.50 & $-9.19(0.05)$ & $163.1(0.1)$ & $-14.89(0.05)$ & $-11.92(0.05)$ & $73.1(0.1)$ & 1 \\
\hline 1989B & 16.47 & $-9.43(0.05)$ & $163.5(0.1)$ & $-14.49(0.05)$ & $-11.72(0.05)$ & $69.7(0.1)$ & 1 \\
\hline 1989B & 17.47 & $-9.31(0.05)$ & $177.4(0.1)$ & $-14.90(0.05)$ & $-12.12(0.05)$ & $66.9(0.1)$ & 1 \\
\hline
\end{tabular}

Notes.

References. (1) Wells et al. 1994; (2) Cristiani et al. 1992; (3) Leibundgut et al. 1991; (4) Mazzali et al. 1993; (5) Blondin \& Tonry 2007; (6) Gómez \& López 1998; (7) Jeffery et al. 1992; (8) Mazzali et al. 1995; (9) Turatto et al. 1996; (10) Leibundgut et al. 1993; (11) Kirshner et al. 1993; (12) Patat et al. 1996; (13) SUSPECT, no reference; (14) S. Blondin et al. 2012, in preparation; (15) Salvo et al. 2001; (16) Li et al. 1999; (17) Turatto et al. 1998; (18) Matheson et al. 2008; (19) Branch et al. 2003; (20) Jha et al. 1999b; (21) Blondin et al. 2011b; (22) Garavini et al. 2004; (23) Garavini et al. 2005; (24) Unknown; (25) Garnavich et al. 2004; (26) Hamuy et al. 2002; (27) Valentini et al. 2003; (28) Krisciunas et al. 2011; (29) Sauer et al. 2008; (30) Wang et al. 2003; (31) Benetti et al. 2004; (32) Pignata et al. 2008; (33) Kotak et al. 2005; (34) Elias-Rosa et al. 2006b; (35) Stanishev et al. 2007; (36) Anupama et al. 2005; (37) Leloudas et al. 2009; (38) Krisciunas et al. 2007; (39) Altavilla et al. 2007; (40) Pastorello et al. 2007; (41) Taubenberger et al. 2008; (42) Wang et al. 2009b; (43) Garavini et al. 2007b; (44) Quimby et al. 2006b; (45) Kasliwal et al. 2008.

(This table is available in its entirety in a machine-readable form in the online journal. A portion is shown here for guidance regarding its form and content.)

wavelength of maximum absorption in the smoothed spectrum is used to determine the velocity (see Blondin et al. $2011 \mathrm{~b}$ for details). When possible the spectra are used to measure the FWHM and pEW for the features as well (see Blondin et al. 2011b for details). The measurements for each spectrum are listed in Table 2. The reference for each spectrum is listed in Table 2, but measurements in all cases were obtained by S. Blondin et al. (2012, in preparation).

\subsubsection{Literature Spectral Sample}

Many SN Ia spectra obtained by various groups using various telescopes, instruments, and reduction methods have been published over time. Although the fidelity of the spectra may not be high or uniform as for spectra from the CfA sample, these data are useful for expanding our sample. When publicly available, usually through the SUSPECT database, ${ }^{4}$ and if the spectra cover the appropriate wavelength ranges, $v_{\mathrm{SiII}}$, $\mathrm{pEW}(\mathrm{Si} \mathrm{II}), v_{\mathrm{CaH} \& \mathrm{~K}}$, and $\mathrm{pEW}(\mathrm{Ca}$ II $)$ have been measured for these SNe. Since the flux calibration is not critical for the velocity measurements, we expect the largest errors to be from poor wavelength calibration; however, the accuracy necessary for our measurements is easily obtained even if mistakes are made while observing or in the reduction process. Comparison of multiple spectra from the same SN both within a data set and with the CfA sample shows that the measurements are consistent within the measurement uncertainty (typically $100 \mathrm{~km} \mathrm{~s}^{-1}$ ). The $\mathrm{pEW}$ measurements are more affected by poor flux calibration. We have examined the individual spectra and have rejected spectra where we believe that the flux calibration is poor. The measurements for each spectrum are listed in Table 2.

Between the CfA sample and Literature sample, there are $1630 v_{\mathrm{Si} \text { II }}, 1630 \mathrm{pEW}\left(\mathrm{Si}\right.$ II), $1192 v_{\mathrm{CaH} \& \mathrm{~K}}$, and $1234 \mathrm{pEW}(\mathrm{Ca}$ II) measurements for $255,255,192$, and $211 \mathrm{SNe}$ Ia, respectively. For the majority of this study, we will restrict our sample to $\mathrm{SNe}$ with $1 \leqslant \Delta m_{15}(B) \leqslant 1.5 \mathrm{mag}$ to match the final sample criteria of FK11. With this restriction, there are 939, 939, 685, and 708 measurements for 141, 141, 109, and $119 \mathrm{SNe}$, respectively.

\footnotetext{
4 http://suspect.nhn.ou.edu/ suspect/
} 
Table 3

Data for Telegraphic Supernovae

\begin{tabular}{lllccrrc}
\hline \hline SN & $\begin{array}{c}v_{\text {Si II }} \\
\left(\mathrm{km} \mathrm{s}^{-1}\right)\end{array}$ & \multicolumn{1}{c}{ UT Date } & $\begin{array}{c}\text { Spec. } \\
\text { Ref. }^{\mathrm{a}}\end{array}$ & $\begin{array}{c}\text { MJD } \\
\text { of } t_{B_{\max }}\end{array}$ & $\begin{array}{c}t_{B_{\max }} \\
\text { Ref. }^{\mathrm{b}}\end{array}$ & $\begin{array}{r}\text { Phase } \\
(\text { days })\end{array}$ & $\begin{array}{c}v_{\text {Si II }}^{0} \\
\left(\mathrm{~km} \mathrm{~s}^{-1}\right)\end{array}$ \\
\hline $1992 \mathrm{ag}$ & -11.8 & 19920703.042 & 1 & 48807.15 & 1 & -0.6 & -11.7 \\
$1992 \mathrm{al}$ & -11.7 & 19920729.3 & 2 & 48838.24 & 1 & -5.7 & -11.3 \\
$1999 \mathrm{cp}$ & -14.1 & 19990619.2 & 3 & 51363.27 & 1 & -15.2 & $\ldots$ \\
$1999 \mathrm{dk}$ & -16.2 & 19990815.06 & 4 & 51413.33 & 1 & -9.4 & $\ldots$ \\
$2000 \mathrm{ca}$ & -11.0 & 20000429.3 & 5 & 51666.63 & 1 & -3.1 & -10.8 \\
$2000 \mathrm{dr}$ & -12.5 & 20001008.1 & 6 & 51833.98 & 2 & -8.3 & $\ldots$ \\
$2001 \mathrm{ba}$ & -10.9 & 20010430.17 & 7 & 52034.36 & 1 & -4.7 & -10.6 \\
$2001 \mathrm{cj}$ & -10.5 & 20010601 & 8 & 52066.04 & 2 & -4.4 & -10.4 \\
$2001 \mathrm{dl}$ & -10.0 & 20010808 & 9 & 52131.47 & 2 & -1.9 & -9.9 \\
$2003 \mathrm{gt}$ & -11.0 & 20030805 & 10 & 52862.17 & 2 & -5.6 & -10.7 \\
$2005 \mathrm{de}$ & -13.3 & 20050804.34 & 11 & 53598.85 & 2 & -12.2 & $\ldots$ \\
$2005 \mathrm{~ms}$ & -13.3 & 20051231 & 12 & 53743.45 & 1 & -7.7 & $\ldots$ \\
$2006 \mathrm{dm}$ & -12.0 & 20060705.1 & 13 & 53928.96 & 2 & -7.3 & $\ldots$ \\
$2006 \mathrm{ef}$ & -12.0 & 20060824 & 14 & 53967.95 & 1 & 1.5 & -12.2 \\
$2006 \mathrm{ej}$ & -13.0 & 20060824 & 14 & 53973.09 & 1 & -4.7 & -12.5 \\
$2006 \mathrm{en}$ & -10.2 & 20060828.9 & 15 & 53971.66 & 1 & 3.7 & -10.4 \\
$2006 \mathrm{os}$ & -12.7 & 20061122.15 & 16 & 54061.68 & 1 & -0.2 & -12.7 \\
$2006 \mathrm{qo}$ & -11.1 & 20061201 & 17 & 54081.96 & 1 & -11.1 & $\ldots$ \\
$2006 \mathrm{td}$ & -10.9 & 20061228.77 & 18 & 54098.92 & 1 & -1.4 & -10.8 \\
$2007 \mathrm{O}$ & -10.0 & 20070122 & 19 & 54123.80 & 1 & -1.3 & -10.0 \\
$2007 \mathrm{sr}$ & -12.1 & 20071220.22 & 20 & 54448.34 & 1 & 6.1 & -12.9 \\
$2008 \mathrm{dt}$ & -14.0 & 20080701.21 & 21 & 54646.73 & 2 & 1.7 & -14.3 \\
$2008 \mathrm{ec}$ & -12.6 & 20080716.08 & 22 & 54674.28 & 2 & -10.6 & $\ldots$ \\
\hline
\end{tabular}

Notes.

a Spectroscopy references: (1) Maza et al. 1992; (2) McNaught et al. 1992; (3) Jha et al. 1999a; (4) Salvo et al. 1999; (5) Aldering \& Conley 2000; (6) Suntzeff \& Smith 2000; (7) Nugent \& Wang 2001; (8) Wang 2001; (9) Patat et al. 2001; (10) Filippenko et al. 2003; (11) Foley et al. 2005; (12) Leonard 2005; (13)Selj et al. 2006; (14) Foley et al. 2006; (15) Elias-Rosa et al. 2006a; (16) Quimby et al. 2006a; (17) Silverman et al. 2006; (18) Gurugubelli et al. 2006; (19) Silverman et al. 2007; (20) Umbriaco et al. 2007; (21) Blondin \& Berlind 2008; (22) Harutyunyan et al. 2008.

${ }^{b}$ Photometry references: (1) Hicken et al. 2009a; (2) Ganeshalingam et al. 2010.

${ }^{\mathrm{c}}$ Uncertainty set to $300 \mathrm{~km} \mathrm{~s}^{-1}$, which is slightly larger than our expected uncertainty for Literature $\mathrm{SNe}$ and larger than the median uncertainty for the F11 sample.

\subsubsection{Telegraphic Spectral Sample}

Despite the CfA sample being quite large, there are several SNe in the CfA3, LOSS, and W09 photometry samples that do not have a CfA or publicly available spectrum (or one with $-6 \leqslant t \leqslant 10$ days). However, every SN in these samples was spectroscopically classified in the IAU Circulars or CBETs. In these reports, the date of observation is always reported, and for most $\mathrm{SNe}$ Ia classified near maximum brightness, $v_{\mathrm{Si} \text { II }}$ is reported. Although these measurements may not be of the same quality - and are certainly not systematically measured in the same way, as we have done with the CfA and public spectra-the uncertainty in the velocity measurement should be relatively small. These additional measurements from the Circulars and Telegrams can significantly expand our final sample.

For most $\mathrm{SNe}$, we are able to determine the phase of each spectrum using the time of maximum reported by Hicken et al. $(2009 b)^{5}$ and Ganeshalingam et al. (2010). We present the data for these $\mathrm{SNe} /$ spectra in Table 3.

\subsubsection{F11 Sample}

The intersection of the CfA, Literature, and Telegraphic spectral samples with the LOSS and CfA3 photometric samples is the sample used for the analysis here. Similar to FK11, we exclude SN 2006bt because of its peculiar nature (Foley

\footnotetext{
5 These values are reported at http://www.cfa.harvard.edu/supernova/ CfA3/sn.tBmax.mlcs17.txt.
}

et al. 2010). We also exclude from our final analysis $\mathrm{SNe}$ with $E(B-V)_{\mathrm{MW}}>0.5 \mathrm{mag}$, corresponding to SNe $1999 \mathrm{ek}$ $\left(E(B-V)_{\mathrm{MW}}=0.561 \mathrm{mag}\right)$ and $2006 \mathrm{ff}\left(E(B-V)_{\mathrm{MW}}=\right.$ $0.954 \mathrm{mag}$ ). Both W09 and FK11 excluded SN $20061 \mathrm{f}$ from their analyses. We call the final sample of $\mathrm{SNe}$ with photometric and spectroscopic measurements the "F11" sample.

\section{VELOCITY GRADIENTS AND MAXIMUM-LIGHT EJECTA VELOCITY}

The temporal coverage of the F11 spectra (in particular, the CfA data) provides an ideal sample for measuring velocity evolution with time. Previous studies have examined relatively small samples of nearby SNe Ia and their spectra to determine velocity gradients for those $\mathrm{SNe}$ (e.g., Benetti et al. 2005; Hachinger et al. 2006). Additionally, ejecta velocities have been measured for some high- $z$ SNe Ia (Hook et al. 2005; Blondin et al. 2006; Bronder et al. 2008; Nordin et al. 2011a; Konishi et al. 2011). W09 separated their sample into two subsamples based on ejecta velocity near maximum brightness. Since $\mathrm{SNe}$ Ia with high velocities near maximum brightness tend to have high velocity gradients, this separation is similar to separating based on velocity gradient.

Although most studies of velocity gradients have focused on $\mathrm{Si}$ II $\lambda 6355$, other features have been examined to some degree. While certain features appear to have little variation from SN to SN (e.g., S II; Pignata et al. 2008), Ca II H\&K velocity near maximum brightness varies significantly among 


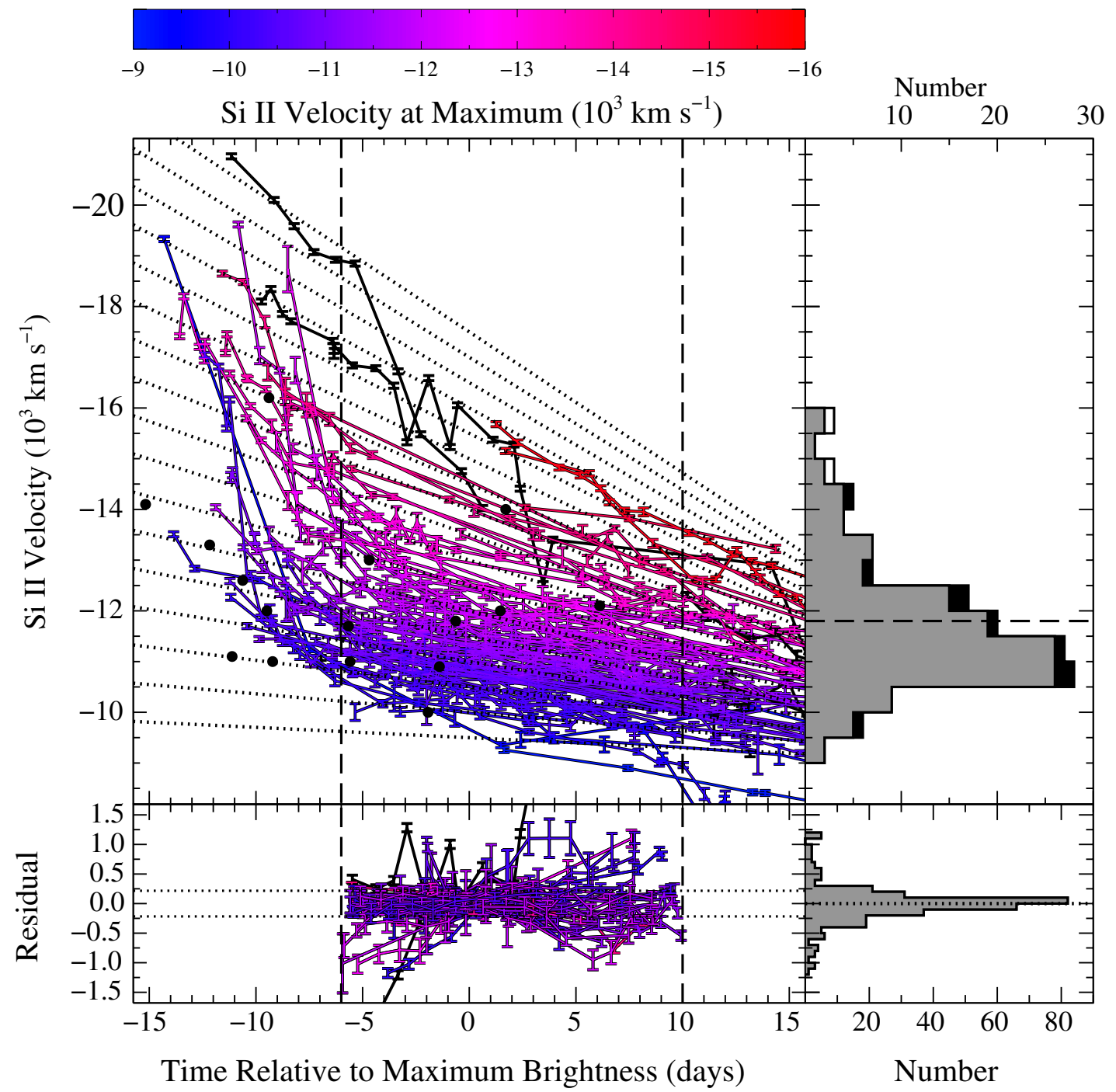

Figure 1. Top left: temporal evolution of Si II $\lambda 6355$ velocity, $v_{\mathrm{Si} \text { II }}$, for the F11 sample of SN Ia with $1 \leqslant \Delta m_{15}(B) \leqslant 1.5$ mag. Measurements for the same SN are connected by solid lines. The vertical dashed lines mark the time interval over which we fit the velocity evolution. The dotted lines represent a subset of the family of functions that describe the velocity evolution (Equation (4)). Each SN is color-coded based on the estimate of $v_{\mathrm{Si}}^{0}$ (using Equation (5)) from the measurement closest to $t=0$ days. The color bar at the top shows how the colors correspond to $v_{\mathrm{Si}}^{0}$. SNe with no measurement in $-6 \leqslant t \leqslant 10$ days are plotted in black. SNe $2003 \mathrm{~W}$ and 2004dt are plotted in black and are excluded from further analysis. Individual measurements from the Telegraphic sample are presented as black points (see Section 2.2.3). Top right: histograms of $v_{\mathrm{Si} \text { II }}^{0}$. The gray histogram is for $\mathrm{SNe}$ where we have measured velocities from spectra. The empty histogram represents $\mathrm{SNe} 2003 \mathrm{~W}$ and 2004dt. The black histogram represents the SNe from the Telegraphic sample. The horizontal dashed line represents the velocity which roughly separated the W09 sample into "Normal" and "High-Velocity" SNe. Bottom left: residuals of $v_{\mathrm{Si} \text { II }}^{0}$ estimates relative to measured velocity within 1 day of $t=0$ days. The dotted horizontal lines represent the standard deviation of $220 \mathrm{~km} \mathrm{~s}^{-1}$. Bottom right: histogram of the residuals. The dotted horizontal line is at 0 .

(A color version of this figure is available in the online journal.)

SNe Ia. Additionally, Ca II H\&K is a strong, blue feature with a large velocity gradient. Using the model spectra and light curves of Kasen \& Plewa (2007), FK11 found that the maximum-brightness velocity of Ca II H\&K strongly correlated with maximum-light color. Ca II $\mathrm{H} \& \mathrm{~K}$ is an excellent candidate for an alternative way to determine intrinsic color.

\subsection{Si II $\lambda 6355$}

In Figure 1, the Si II velocity is shown as a function of phase relative to $B$ maximum for the $141 \mathrm{SNe}$ Ia with a velocity measurement, a time of maximum, and $1 \leqslant \Delta m_{15}(B) \leqslant 1.5 \mathrm{mag}$. The $\mathrm{SNe}$ with higher velocity near maximum brightness also have larger velocity gradients. However, at very early times $(t \lesssim-10$ days), some low velocity gradient $\mathrm{SNe}$ have similar velocities to high velocity gradient SNe. Similarly, at later times $(t \gtrsim 15$ days), it is difficult to distinguish $\mathrm{SNe}$ with different ve- locity gradients with a single velocity measurement. Near maximum brightness, the velocity evolution is close to linear in time.

There are two SNe, SNe 2003W and SN 2004dt, that are outliers in both early-time velocity and velocity gradient. SN $2004 \mathrm{dt}$ has very high early-time $v_{\mathrm{Si} \text { II }}$. The velocity gradient at early times is consistent with other $\mathrm{SNe}$, but also changes dramatically. It declines by $2750 \mathrm{~km} \mathrm{~s}^{-1}$ in only 1.4 days, which was previously noted (Altavilla et al. 2007). Additionally, the Si II $\lambda 6355$ feature is wide, flat-bottomed, and potentially contains two components (Wang et al. 2006; Altavilla et al. 2007). SN $2004 \mathrm{dt}$ is also a significant outlier in the relation between velocity gradient and nebular velocity shifts (Maeda et al. 2010). Finally, SN 2004dt has extremely high line polarization (Leonard et al. 2005; Wang et al. 2006). In summary, it appears that SN 2004dt is spectroscopically distinct from the majority of SNe Ia and potentially different from even very high velocity SNe. 


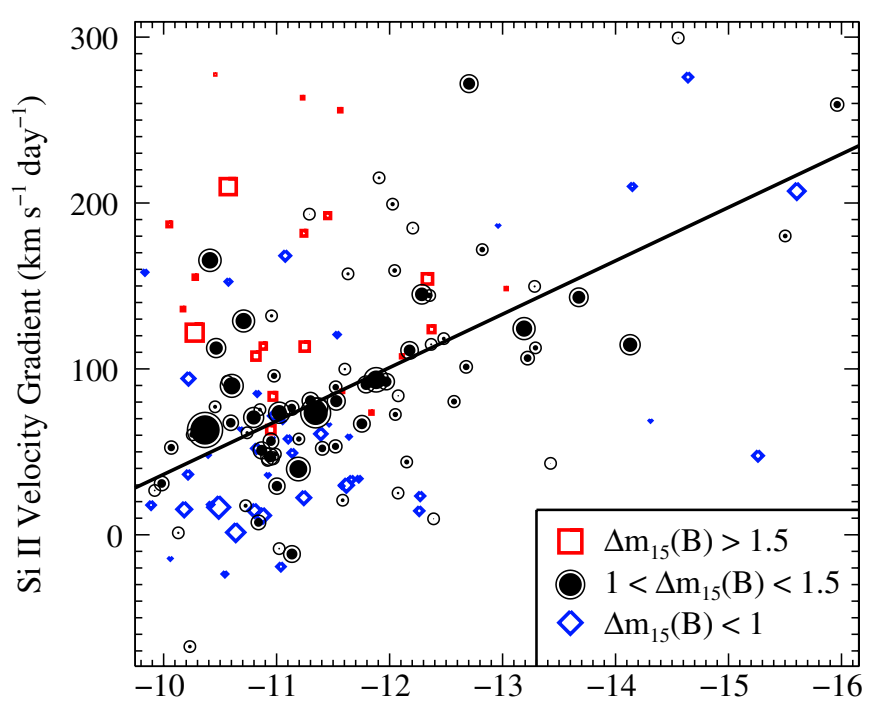

Si II Velocity at Maximum Brightness $\left(10^{3} \mathrm{~km} \mathrm{~s}^{-1}\right)$

Figure 2. Best-fit values to the slope and offset for a line (Equation (1)) describing the $v_{\text {Si II }}$ evolution of the F11 sample. SNe with $\Delta m_{15}<1$, $1 \leqslant \Delta m_{15} \leqslant 1.5$, and $\Delta m_{15}>1.5 \mathrm{mag}$ are represented by blue diamonds, black circles, and red squares, respectively. The size of the points is inversely proportional to the size of the uncertainty. Each point with $1 \leqslant \Delta m_{15} \leqslant 1.5 \mathrm{mag}$ has a circle surrounding it, with a minimum size to help the reader see the smallest points (corresponding to $\mathrm{SNe}$ with the largest uncertainty). The solid line is the best-fit line for the $1 \leqslant \Delta m_{15} \leqslant 1.5 \mathrm{mag}$ subsample.

(A color version of this figure is available in the online journal.)

SN 2003W has both the highest $v_{\text {Si II }}$ measurements of any $\mathrm{SN}$ in the F11 sample, exceeding $-20,000 \mathrm{~km} \mathrm{~s}^{-1}$, and a dramatic velocity decline near maximum light, declining by over $4000 \mathrm{~km} \mathrm{~s}^{-1}$ over 5 days (where the typical value is $100-200 \mathrm{~km} \mathrm{~s}^{-1} \mathrm{day}^{-1}$ ). Its Si II profile appears to be flatbottomed and probably is the result of two strong components. Unfortunately, there never appears to be two distinct components in any of our spectra. Perhaps additional spectroscopy at slightly later times would reveal this. Wang \& Wheeler (2008) report that there is an unpublished epoch of spectropolarimetry for SN 2003W. These data may be extremely informative to understanding the nature of this $\mathrm{SN}$, particularly given the extraordinary polarization of SN 2004dt. Although SN 2003W is clearly a high-velocity and high velocity gradient $\mathrm{SN}$, we have excluded it from further analysis because of its peculiar velocity evolution. It is unclear if SN $2003 \mathrm{~W}$ is a truly peculiar SN or if the velocity evolution of other $\mathrm{SNe}$ Ia with extremely high $v_{\mathrm{Si} \text { II }}$ at early times is similar to that of SN 2003W.

A line was fit to the velocity data for each SN (except for $\mathrm{SNe} 2003 \mathrm{~W}$ and 2004dt) with $\geqslant 3$ measurements in the window $-6 \leqslant t \leqslant 10$ days regardless of $\Delta m_{15}(B)$. We fit for

$$
v(t)=\dot{v} t+v^{0}
$$

where $v(t)$ is the velocity, $t$ is the time relative to $B$ maximum, and $\dot{v}$ and $v^{0}$ are a slope and offset for the line, corresponding to the velocity gradient and velocity at time of $B$ maximum $(t=0)$, respectively. The resulting fit values for $\dot{v}$ and $v^{0}$ are shown in Figure 2.

SNe Ia from the different light-curve shape subsamples have different relations between Si II $\lambda 6355$ velocity and velocity gradient. For SNe Ia with $1 \leqslant \Delta m_{15}(B) \leqslant 1.5 \mathrm{mag}$, there is a clear relation between the two values such that $\mathrm{SNe}$ with a higher velocity at maximum also have a higher velocity gradient. This is quantitative confirmation of the previously noted correlation.
The fast decliners tend to have low velocities, but high velocity gradients relative to the other $\mathrm{SNe}$, confirming previous results (e.g., Benetti et al. 2005). The slow decliners have a large scatter, but also have a similar trend to the moderate decliners (although they may have a slightly lower velocity gradient for a given velocity).

For the moderate decliners, a line was then fit to the values of $\dot{v}$ and $v^{0}$,

$$
\dot{v}=\alpha v^{0}+\beta .
$$

Substituting for $\dot{v}$, we can construct a family of functions that describe the velocity evolution near maximum brightness and only depend on $v^{0}$,

$$
\begin{aligned}
v(t) & =\dot{v} t+v^{0} \\
& =\left(\alpha v^{0}+\beta\right) t+v^{0} \\
& =(1+\alpha t) v^{0}+\beta t
\end{aligned}
$$

Using the fit values, the family of functions correspond to

$$
v_{\mathrm{Si} \text { II }}\left(v_{\mathrm{Si} \mathrm{II}}^{0}, t\right)=v_{\mathrm{Si} \mathrm{II}}^{0}(1-0.0322 t)-0.285 t,
$$

where $v_{\mathrm{Si} \text { II }}^{0}$ is the velocity at maximum, $t$ is measured in days relative to maximum brightness, and $v_{\mathrm{Si} \text { II }}$ and $v_{\mathrm{Si} \text { II }}^{0}$ are measured in $10^{3} \mathrm{~km} \mathrm{~s}^{-1}$. This family of functions describes the velocity evolution of $\mathrm{SNe}$ Ia near maximum brightness, and a number of the functions are plotted in Figure 1.

A test of how well this family of functions performs over our chosen time interval $(-6 \leqslant t \leqslant 10$ days $)$ is to examine the residuals of the functions relative to the data. Every velocity measurement within our time interval provides an estimate of $v_{\mathrm{Si} \text { II }}^{0}$ for that $\mathrm{SN}$. For all $\mathrm{SNe}$ with a velocity measurement within a day of maximum brightness, we measure the "residual": the difference between the predicted $v_{\mathrm{Si} \text { II }}^{0}$ from a single measurement and the measured velocity within 1 day of maximum brightness. We call this the residual, but it is not exactly the residual since the measurements were not obtained exactly at $t=0$ days. The residuals are shown in the bottom-left panel of Figure 1.

The residuals are small and centered on zero. The average for all residuals is $-10 \mathrm{~km} \mathrm{~s}^{-1}$ and the standard deviation is $220 \mathrm{~km} \mathrm{~s}^{-1}$. There are few residuals that are more than $1000 \mathrm{~km} \mathrm{~s}^{-1}$ from zero. The residuals are slightly larger at the extremes of our time interval than near maximum brightness, but the difference is still relatively small. The typical measurement uncertainty is $\lesssim 100 \mathrm{~km} \mathrm{~s}^{-1}$. Velocity uncertainty from hostgalaxy rotation is $\lesssim 300 \mathrm{~km} \mathrm{~s}^{-1}$. Therefore, the scatter in the derived relation is not the dominant source of uncertainty for $v_{\mathrm{Si} \text { II }}^{0}$. Combining these sources of uncertainty, the total uncertainty is $\lesssim 400 \mathrm{~km} \mathrm{~s}^{-1}$.

Using Equation (4) and a single velocity measurement in the time interval $-6 \leqslant t \leqslant 10$ days, an estimate of $v_{\mathrm{Si} \text { II }}^{0}$ can be made for a SN. This allows for a direct comparison of all $\mathrm{SNe}$ with such measurements. Solving Equation (4) for $v_{\mathrm{Si} \text { II }}^{0}$, one finds

$$
v_{\mathrm{Si} \text { II }}^{0}=\left(v_{\mathrm{Si}_{\text {II }}}+0.285 t\right) /(1-0.0322 t) \text {. }
$$

Table 1 lists $v_{\mathrm{Si} \text { II }}^{0}$ for each $\mathrm{SN}$ in the F11 sample with a measurement in $-6 \leqslant t \leqslant 10$ days. The value given is for the measurement closest to $t=0$ days. In Figure 3 , we plot $v_{\mathrm{Si} \text { II }}^{0}$ as a function of $\Delta m_{15}(B)$ for the full F11 sample. We note that these values are derived from Equation (5), and the velocity estimated for SNe with $\Delta m_{15}(B)<1 \mathrm{mag}$ or $\Delta m_{15}(B)>1.5$ mag may 


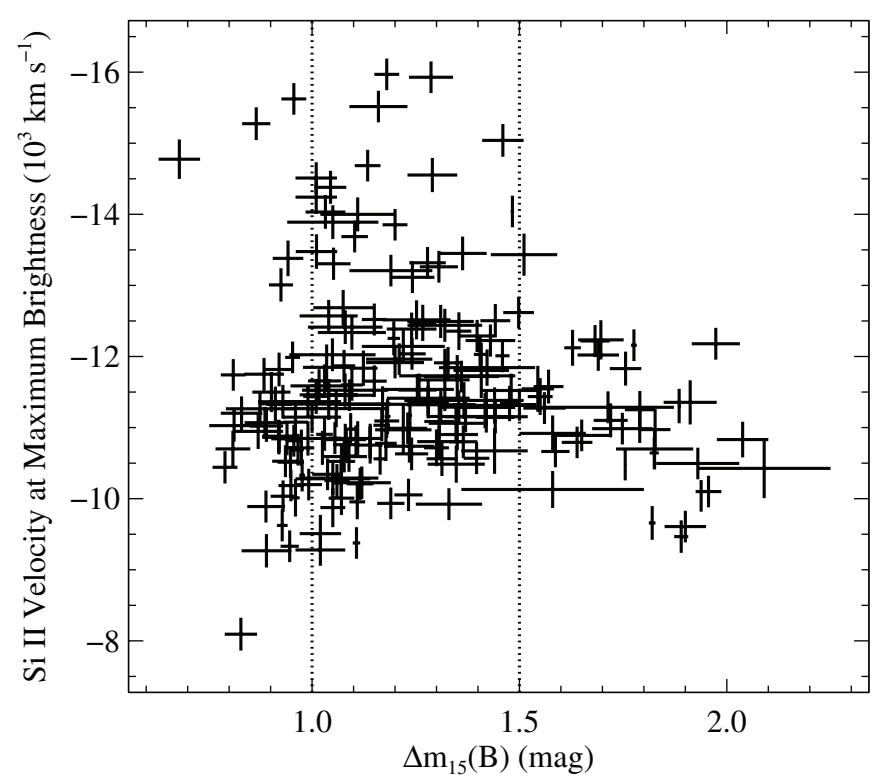

Figure 3. Si II velocity at maximum brightness $\left(v_{\mathrm{Si} \text { II }}^{0}\right)$ as a function of $\Delta m_{15}(B)$ for the F11 sample. The vertical dotted lines mark the region of our full analysis $\left(1 \leqslant \Delta m_{15}(B) \leqslant 1.5 \mathrm{mag}\right)$.

have systematic offsets (but typically $<1000 \mathrm{~km} \mathrm{~s}^{-1}$ ) from their true value. Regardless, there appears to be a dearth of highvelocity/fast-declining SNe Ia, but there are not many SNe with large $\Delta m_{15}(B)$ in the F11 sample. Slow-declining SNe Ia can have high-velocity ejecta, but there also appear to be a significant population of low-velocity/slow-declining SNe Ia.

The lack of high-velocity/fast-declining SNe Ia is not unexpected. SNe Ia with $\Delta m_{15}(B) \gtrsim 1.5 \mathrm{mag}$ are spectroscopically distinct from slower-declining SNe Ia, being spectroscopically similar to SN 1991bg (Filippenko et al. 1992a; Leibundgut et al. 1993). No high-velocity SN 1991bg-like SN Ia has yet been discovered. It is worth noting that despite having a low velocity near maximum brightness, SNe Ia with $\Delta m_{15}(B)>1.5 \mathrm{mag}$ tend to have high velocity gradients (Benetti et al. 2005; Figure 2). Therefore, Equation (4) may not properly describe the velocity evolution of these SNe.

Since fast-declining SNe Ia have ejecta velocities similar to or lower than slower-declining SNe Ia at maximum brightness, one cannot attribute their fast post-maximum decline to a rapidly expanding photosphere (e.g., Höflich et al. 1996; Pinto \& Eastman 2001). Rather, the post-maximum decline is likely set by the amount of Fe-group elements in the ejecta (and thus linked to ${ }^{56} \mathrm{Ni}$ production in the explosion and the $\mathrm{SN}$ peak luminosity; Kasen \& Woosley 2007).

Benetti et al. (2005) also showed a lack of high velocity gradient SNe Ia with $\Delta m_{15}(B)<1 \mathrm{mag}$. Many of these SNe are spectroscopically similar to SN 1991T (Filippenko et al. 1992b), which have a low velocity near maximum brightness (W09). Although the relation between velocity at maximum brightness and velocity gradient for SN 1991T-like SNe appears to be consistent with that found in Equation (4), there are larger residuals for these $\mathrm{SNe}$.

Within the limited range of $1 \leqslant \Delta m_{15}(B) \leqslant 1.5 \mathrm{mag}$, there is no clear trend between $v_{\mathrm{Si} \text { II }}^{0}$ and $\Delta m_{15}(B)$; however, the $\mathrm{SNe}$ with the slowest ejecta are found among the slowest decliners.

In Figure 4, we show the $v_{\mathrm{Si} \text { II }}^{0}$ cumulative distribution functions (CDFs) for SNe Ia in the F11 sample with $\Delta m_{15}(B)<1$, $1 \leqslant \Delta m_{15}(B) \leqslant 1.5$, and $\Delta m_{15}(B)>1.5 \mathrm{mag}$. Using these

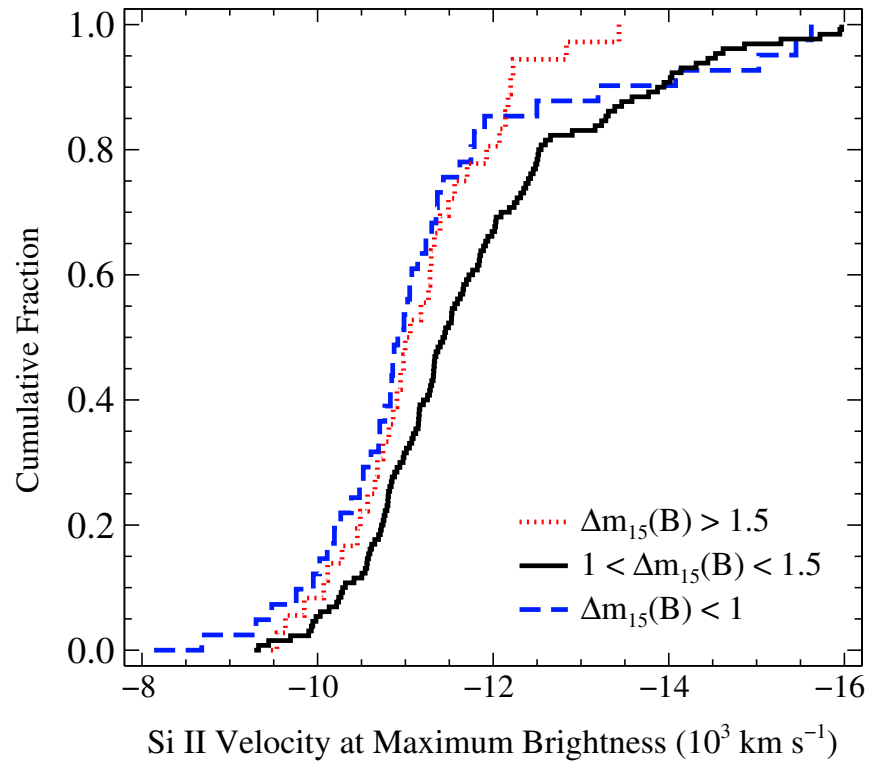

Figure 4. Si II velocity at maximum brightness $\left(v_{\mathrm{Si} \text { II }}^{0}\right) \mathrm{CDF}$ for the F11 sample with $\Delta m_{15}(B)<1,1 \leqslant \Delta m_{15}(B) \leqslant 1.5$, and $\Delta m_{15}(B)>1.5 \mathrm{mag}$ (dashed blue, solid black, and dotted red lines, respectively).

(A color version of this figure is available in the online journal.)

broad groups, it is easy to see the trends described above: there is a lack of high-velocity/fast-declining $\mathrm{SNe}$ Ia, there are a significant number of high-velocity/slow-declining $\mathrm{SNe}$ Ia, and there are a significant number of low-velocity/slowdeclining SNe Ia. Additionally, the figure shows that $\mathrm{SNe}$ Ia with $1 \leqslant \Delta m_{15}(B) \leqslant 1.5 \mathrm{mag}$ tend to have a higher $v_{\mathrm{Si} \text { II }}^{0}$ than $\mathrm{SNe}$ Ia in the other groups. Performing Kolmogorov-Smirnov $(\mathrm{K}-\mathrm{S})$ tests, we find that it is unlikely that $\mathrm{SNe}$ Ia with $1 \leqslant \Delta m_{15}(B) \leqslant 1.5 \mathrm{mag}$ and those with $\Delta m_{15}(B)<1 \mathrm{mag}$ and those with $\Delta m_{15}(B)>1.5 \mathrm{mag}$ come from the same parent population ( $p=0.0065$ and 0.04 , respectively).

Because of the spectroscopic difference between SNe Ia with $1 \leqslant \Delta m_{15}(B) \leqslant 1.5 \mathrm{mag}$ and many of the SNe Ia outside that range, we find it prudent to restrict our analysis to this range. Additionally, the differences in velocity populations for the three groups above and the different relation between velocity and velocity gradient for SN 1991 bg-like SNe Ia (see Figure 2) give further reason to focus on the limited range in light-curve shape. Finally, W09 found that "Normal" and "High-Velocity" SNe Ia have similar light-curve shape and host-galaxy morphology distributions over this range.

Hicken et al. (2009a) compiled morphology classifications for most of the host galaxies of SNe Ia in the F11 sample. Figure 5 presents $v_{\mathrm{Si} \text { II }}^{0}$ as a function of host-galaxy morphology for the F11 sample with $1 \leqslant \Delta m_{15}(B) \leqslant 1.5 \mathrm{mag}$. As found by Wang et al. (2009a), but now using full velocity information, we do not see any significant differences in the Si II velocities observed in host galaxies of different morphologies. Although there are few very low velocity $\left(v_{\mathrm{Si} \text { II }}^{0} \gtrsim-10,000 \mathrm{~km} \mathrm{~s}^{-1}\right)$ SNe Ia in elliptical galaxies, and no high-velocity $\left(v_{\text {Si II }}^{0} \lesssim\right.$ $-11,800 \mathrm{~km} \mathrm{~s}^{-1}$ ) SNe Ia in galaxies later than $\mathrm{Sc}$, the number of SNe in these morphology bins is small. This may come from the combination of a slight trend between $v_{\mathrm{Si} \text { II }}^{0}$ and $\Delta m_{15}(B)$ (Figure 3) as well as faster declining SNe Ia having a higher frequency in early-type galaxies (e.g., Howell 2001).

We also examine the $B_{\max }-V_{\max }$ CDF for separate $v_{\mathrm{Si} \text { II }}^{0}$ bins. Figure 6 shows these CDFs for four $v_{\mathrm{Si} \text { II }}^{0}$ bins $\left(v_{\mathrm{Si} \text { II }}^{0}<-10,800\right.$, 


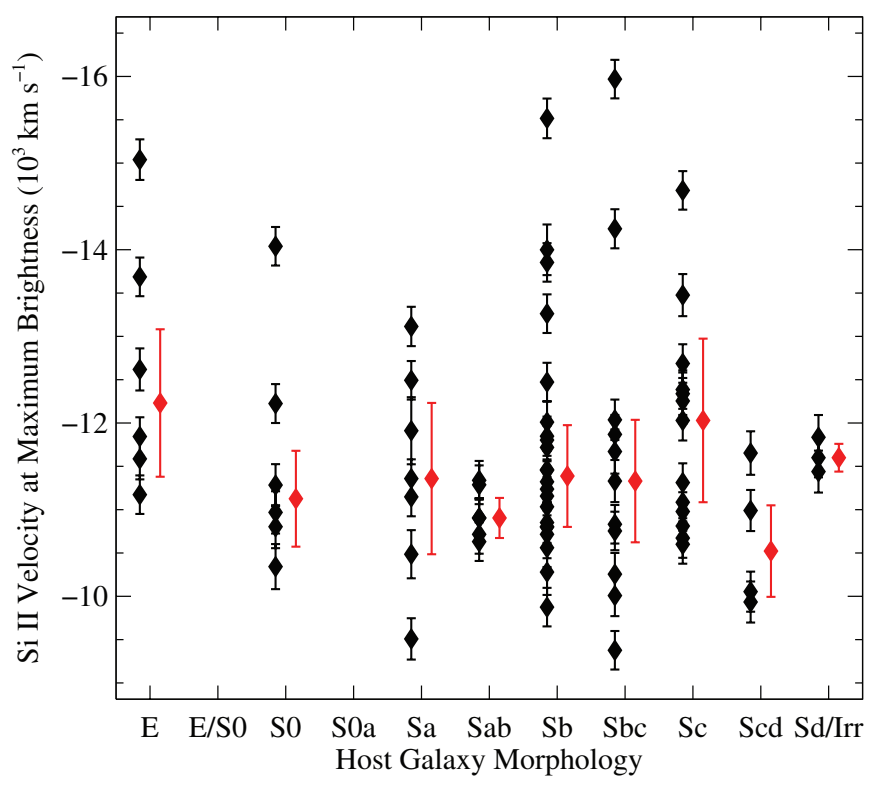

Figure 5. Si II velocity at maximum brightness $\left(v_{\mathrm{Si} \text { II }}^{0}\right)$ as a function of hostgalaxy morphology for the F11 sample with $1 \leqslant \Delta m_{15}(B) \leqslant 1.5 \mathrm{mag}$. The black circles represent individual $\mathrm{SNe}$, while the red diamonds represent the median value for each morphology bin with the error bars representing the median absolute deviation for each morphology bin.

(A color version of this figure is available in the online journal.)

$-10,800>v_{\mathrm{Si} \text { II }}^{0}>-11,800,-11,800>v_{\mathrm{Si} \text { II }}^{0}>-12,800$, and $v_{\mathrm{Si} \text { II }}^{0}>-12,800 \mathrm{~km} \mathrm{~s}^{-1}$, corresponding to $21,31,17$, and $15 \mathrm{SNe}$, respectively). There is a general trend for the median color to become redder with velocity. While the highest and lowest velocity bins have similar shapes and appear to simply be offset in color space, the middle two velocity bins have different shapes. The second velocity bin $\left(-10,800>v_{\mathrm{Si}_{\text {II }}}^{0}>\right.$ $-11,800 \mathrm{~km} \mathrm{~s}^{-1}$ ) has a broad distribution of colors, containing the bluest $\mathrm{SNe}$ and a significant population of highly reddened $\mathrm{SNe}$. Meanwhile, the third velocity bin $\left(-11,800>v_{\mathrm{Si} \text { II }}^{0}>\right.$ $-12,800 \mathrm{~km} \mathrm{~s}^{-1}$ ) has a relatively narrow range of colors and not as many very red $\mathrm{SNe}$ as the second velocity bin. Performing $\mathrm{K}-\mathrm{S}$ tests, we find that the parent population of the lowest velocity bin is significantly different from the other velocity bins (all with probability $<0.04$ ). Despite the apparently different shapes, K-S tests do not find a statistically significant difference between any of the other velocity bins.

\section{2. $C a I I H \& K$}

In this section, we repeat much of the same analysis presented in Section 3.1, but for the CaII H\&K feature. As noted in Section 1 , Ca II H\&K is a very strong feature that is relatively blue and thus visible in optical spectra at $z>1$ and shows significant velocity evolution and diversity. These qualities make Ca II H\&K appealing for use as an intrinsic color indicator at high $z$.

However, unlike $\mathrm{Si}$ II $\lambda 6355$, Ca II $\mathrm{H} \& \mathrm{~K}$ is in a complicated spectral region. Whereas Si II $\lambda 6355$ is a single, isolated feature, other lines are blended with $\mathrm{Ca} I \mathrm{II} \& \mathrm{~K}$, and the pseudocontinuum near the feature is set by a combination of line blanketing and nearby strong features. Furthermore, Ca II H\&K often shows a complicated absorption profile, with a flat bottom, shoulders, and/or multiple distinct absorption minima, complicating any analysis (Figure 7).

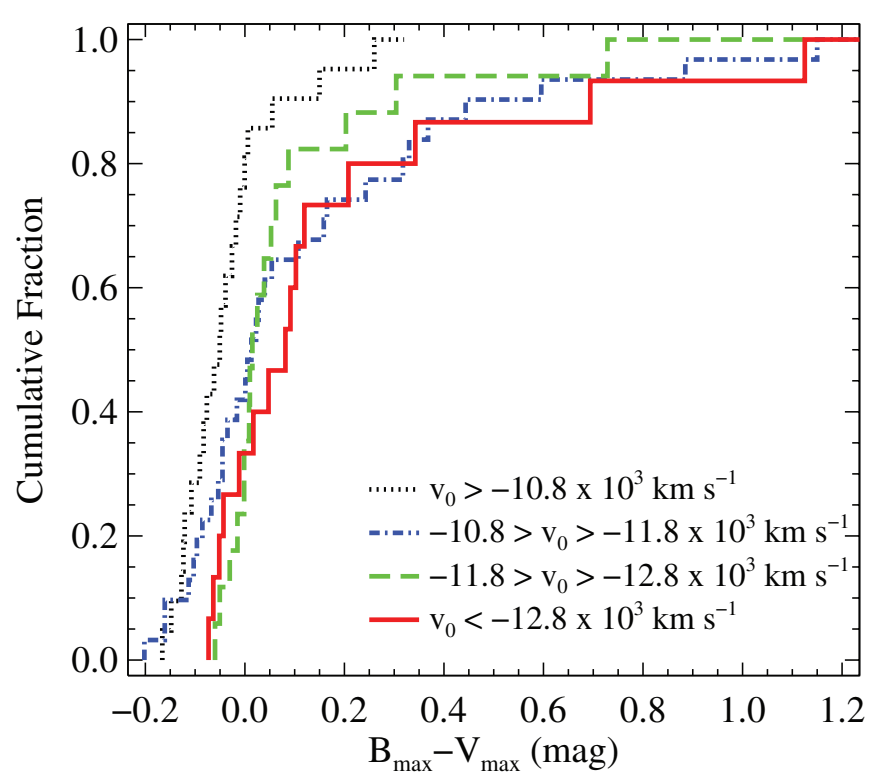

Figure 6. $B_{\max }-V_{\max }$ CDFs for the F11 sample with $v_{\mathrm{Si}_{\text {II }}}^{0}>-10.8,-10.8>$ $v_{\mathrm{Si} \text { II }}^{0}>-11.8,-11.8>v_{\mathrm{Si} \text { II }}^{0}>-12.8$, and $v_{\mathrm{Si} \text { II }}^{0}<-12.8 \times 10^{3} \mathrm{~km} \mathrm{~s}^{-1}$ (dotted black, dot-dashed blue, dashed green, and solid red lines, respectively). W09 set $-11,800 \mathrm{~km} \mathrm{~s}^{-1}$ as the dividing line between the two velocity groups outlined in that work.

(A color version of this figure is available in the online journal.)

The velocity of each absorption minimum in the Ca II H\&K feature of the smoothed spectra is automatically recorded ( $\mathrm{S}$. Blondin et al. 2012, in preparation). We only examine spectra with one or two minima. If two minima are found, the higher/ lower velocities are classified as "blue"/"red." If only one minimum is found, it is categorized as the red or lowervelocity component. In Figure $8, v_{\mathrm{CaH} \& \mathrm{~K}}$ found from the blue and red absorption minima are shown as a function of $v_{\mathrm{Si} \text { II }}$. Examples can be seen in Figure 7. The two absorptions show distinct "clouds" representing a clear correlation between $v_{\text {Si II }}$ and $v_{\mathrm{CaH} \& \mathrm{~K}}$ for each component independently. However, there are several red velocity measurements which overlap with the blue cloud of points. The most likely explanation is that these measurements correspond to a higher velocity component that was incorrectly identified as a red component. The obvious reason for this is if the blue component dominates over the red component to the point where there is no distinct minimum for the redder component.

Our goal of connecting velocities and colors will be undermined if we misidentify the absorption components. When $v_{\mathrm{Si} \text { II }}$ has been measured, there is no ambiguity, but for $z>0.4, v_{\text {Si II }}$ is rarely measurable. To remove interlopers from our Ca II H\&K velocities, we examine four categories of objects with the properties: $f_{\mathrm{Ca}}=f_{\lambda}\left(v_{\mathrm{CaH} \& \mathrm{~K}}+9000 \mathrm{~km} \mathrm{~s}^{-1}\right) / f_{\lambda}\left(v_{\mathrm{CaH} \& \mathrm{~K}}\right)<1.5$, $\mathrm{Ca}$ II $\mathrm{H} \& \mathrm{~K}$ feature has a visible red shoulder, $\mathrm{pEW}(\mathrm{Ca}$ II $)>$ $150 \AA$, and $v_{\mathrm{CaH} \& \mathrm{~K}}>-14,000 \mathrm{~km} \mathrm{~s}^{-1}$.

A ratio of fluxes in the spectra at $v_{\mathrm{CaH} \& \mathrm{~K}}$ and offset by $+9000 \mathrm{~km} \mathrm{~s}^{-1}$, which we call $f_{\mathrm{Ca}}$, is a strong discriminant. If $v_{\mathrm{CaH} \& \mathrm{~K}}$ is matched to a blue feature, the flux ratio may be small if there is an additional absorption component to the red. Conversely, a red feature will generally have much higher flux $9000 \mathrm{~km} \mathrm{~s}^{-1}$ redward of the absorption. One of us (R.J.F.) visually inspected (multiple inspections were performed for all spectra, with at least three classifications per spectrum, and requiring consistent results) all spectra to determine qualitative characteristics of the spectra. After making all qualitative 


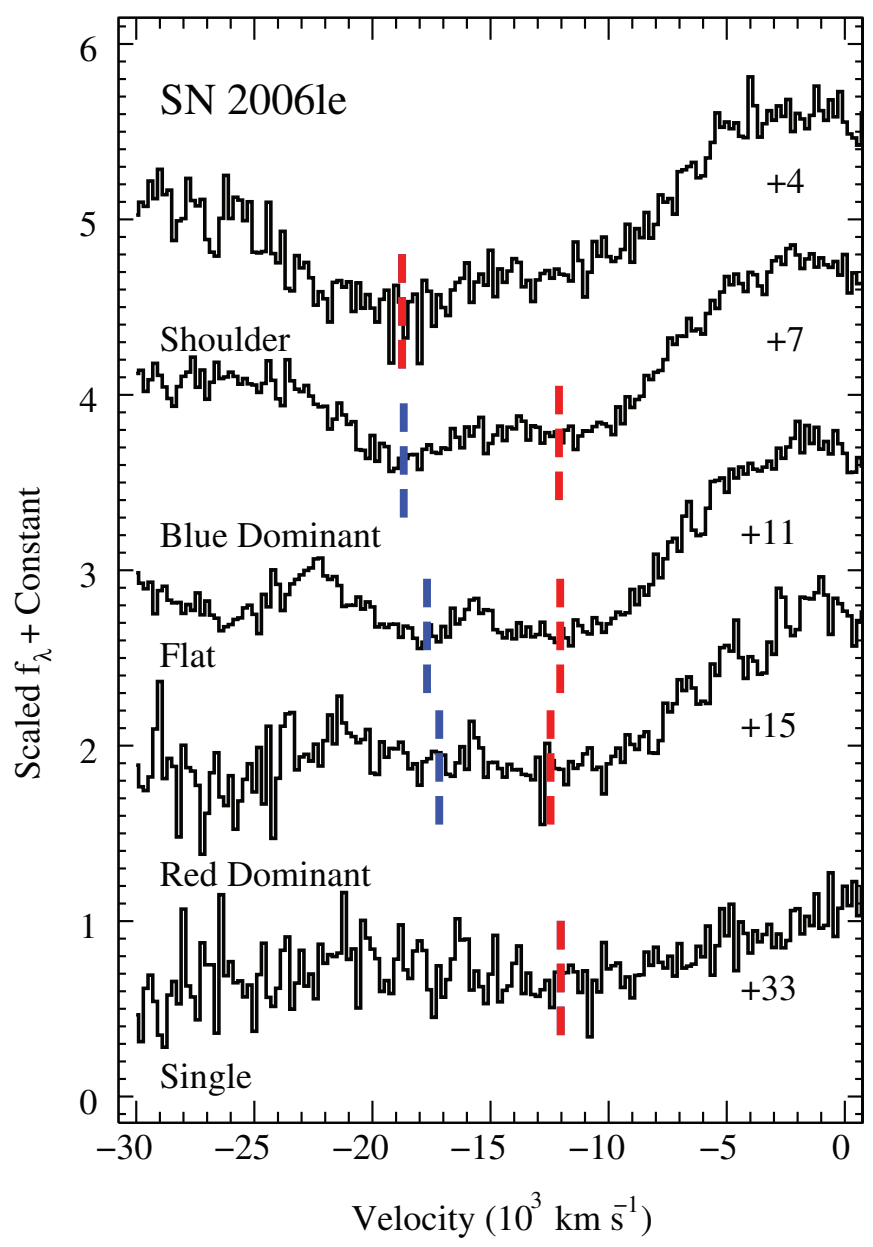

Figure 7. Optical spectra of SN 2006le showing the CaII H\&K feature in velocity space relative to $3945 \AA$. Their phase is marked to the right. The blue and red absorption velocities are shown as dashed blue and red vertical lines, respectively. The spectra show the range of possible spectral shapes (from top to bottom): a red shoulder that the automatic procedure does not detect, causing the blue feature to be classified as red, a double-absorption profile where the blue component is stronger, a double-absorption profile where the two components are of comparable strength, a double-absorption profile where the red component is stronger, and a single component where no blue absorption is found.

(A color version of this figure is available in the online journal.)

assessments, it was found that those with a noticeable red shoulder on the feature were exclusively in the blue cloud (Figure 8). These spectra correspond to the case where the blue component is dominant and the automatic procedure could not identify the other component. Additionally, we found that spectra with a large $\mathrm{pEW}(\mathrm{Ca}$ II) generally overlapped with the blue cloud; however, many of these spectra were at the highest velocities (for both $\mathrm{Ca}$ II and $\mathrm{Si}$ II), and their true association was ambiguous. Since these spectra were also typically at the earliest phases ( $t \lesssim-7$ days), excluding them will make little difference to our final analysis. We therefore chose to include these spectra. Finally, very few blue absorption features are at $v_{\mathrm{CaH} \& \mathrm{~K}}>14,000 \mathrm{~km} \mathrm{~s}^{-1}$. We therefore declare any red $v_{\mathrm{CaH} \& \mathrm{~K}}$ with a velocity below this threshold to be a true measurement of the red ejecta velocity regardless of other measurements. Ultimately, spectra with $v_{\mathrm{CaH} \& \mathrm{~K}}<-14,000 \mathrm{~km} \mathrm{~s}^{-1}$ and either if $f_{\mathrm{Ca}}<1.5$ or if there was a visible red shoulder, are assumed to be a misidentification and were rejected. Graphically, these samples can be seen in Figure 8.

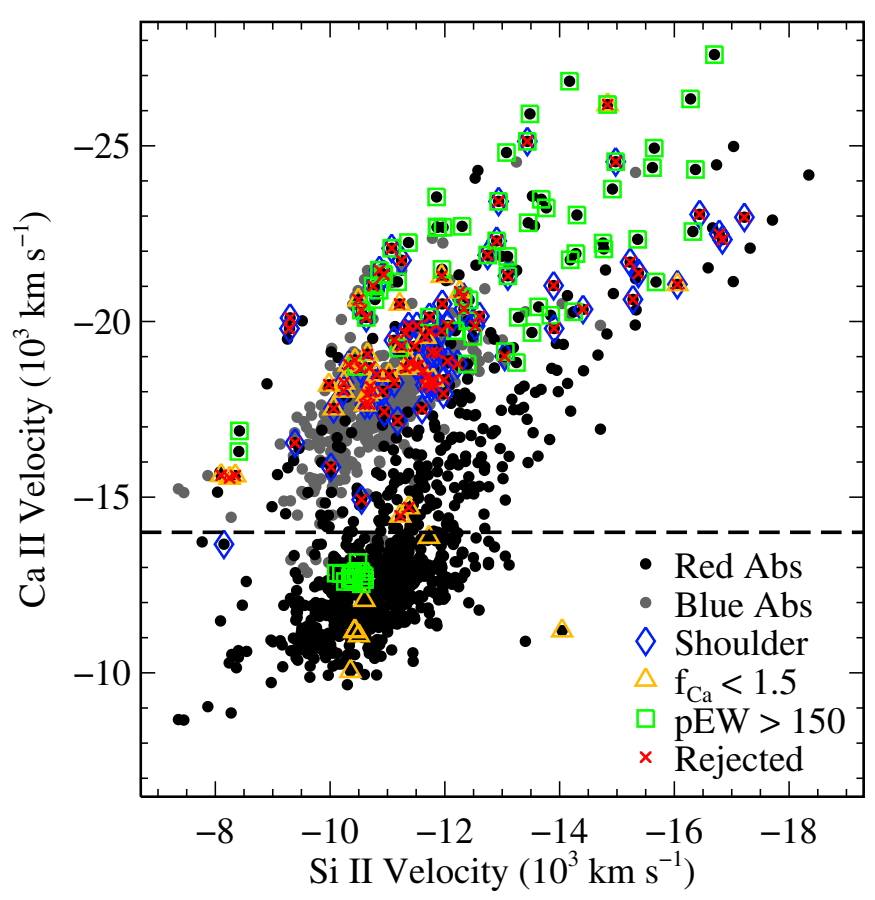

Figure 8. $v_{\mathrm{CaH} \& \mathrm{~K}}$ as a function of $v_{\mathrm{Si} \text { II }}$. The gray and black points correspond to the blue and red absorption velocities, respectively. The green squares, gold triangles, and blue diamonds correspond to spectra with $\mathrm{pEW}(\mathrm{Ca}$ II) $>150 \AA, f_{\mathrm{Ca}}<1.5$, and having a visible red shoulder (e.g., Figure 7), respectively. The dashed line represents a velocity of $-14,000 \mathrm{~km} \mathrm{~s}^{-1}$. Red absorption measurements with a visible red shoulder or $f_{\mathrm{Ca}}<1.5$ and with $v_{\mathrm{CaH} \& \mathrm{~K}}<-14,000 \mathrm{~km} \mathrm{~s}^{-1}$ have been rejected from our analysis and are marked with a red "X."

(A color version of this figure is available in the online journal.)

In Figure 9, the red Ca II velocity is shown as a function of phase relative to $B$ maximum for the 685 spectra of 109 $\mathrm{SNe}$ Ia with a Ca II $\mathrm{H} \& \mathrm{~K}$ measurement, a time of maximum, and $1 \leqslant \Delta m_{15}(B) \leqslant 1.5 \mathrm{mag}$. Only spectra which have passed our previous cuts are shown. The velocity evolution of Ca II is similar to that of Si II, but Ca II is typically at a higher velocity at a given epoch (see Figure 8).

There are fewer spectra and fewer $\mathrm{SNe}$ with a $v_{\mathrm{CaH} \& \mathrm{~K}}$ measurement than for $v_{\mathrm{Si} \text { II }}$. This is because (1) the CfA FAST spectra extend only to $\sim 3500 \AA$ in the blue, and the Ca II H\&K absorption minimum is often blueward of these spectra, and (2) the above procedure rejected many spectra. Regardless, the same trend that higher-velocity $\mathrm{SNe}$ have higher velocity gradients is clear. This is also shown in Figure 10, which is similar to Figure 2.

It appears that early-time ( $t<-6$ days) Ca II H\&K data might be a cleaner diagnostic of maximum-light velocity and velocity gradient than for Si II $\lambda 6355$, but the small number of spectra at these phases prevents a definitive statement. This might be the result of inspecting and rejecting individual spectra for Ca II H\&K and not for Si II $\lambda 6355$, which might also have multiple components at early phases. SNe with high-velocity Ca II H\&K features continue to have higher velocity than their lower velocity counterparts to later phases than is seen with Si II $\lambda 6355$. Despite this, the linear velocity evolution does not maintain at these later phases (there appears to be a plateau for most $\mathrm{SNe}$ ). As a result of a paucity of early-time data and the changing velocity gradient at later times, we define the phase range of $-4<t<9$ days to be the range over which $v_{\mathrm{CaH} \& \mathrm{~K}}^{0}$ can be cleanly measured. 


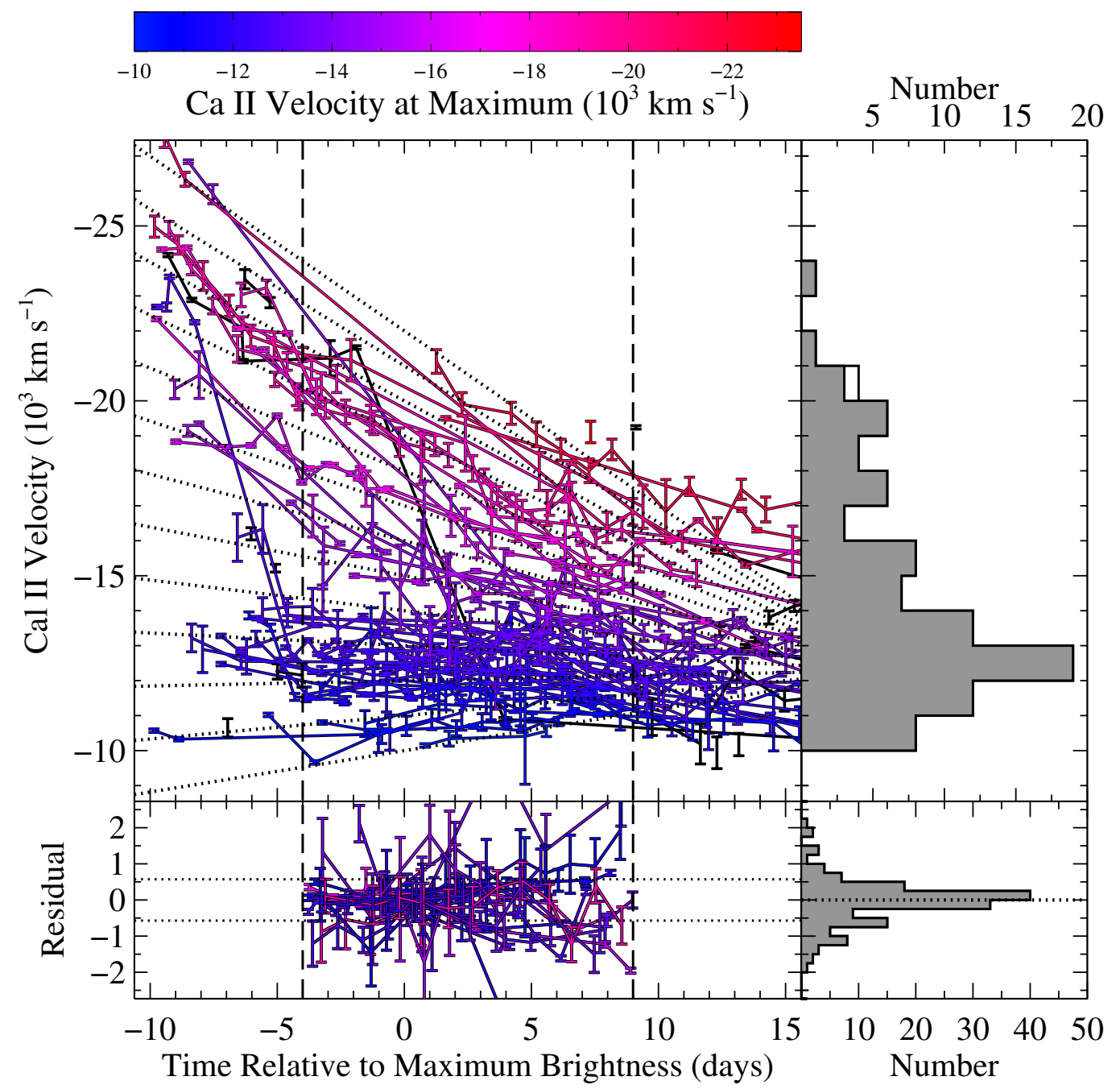

Figure 9. Same as Figure 1, except for $v_{\mathrm{CaH} \& \mathrm{~K}}$.

(A color version of this figure is available in the online journal.)

Performing the same procedure as above (again, ignoring SN 2004dt, which also has peculiar Ca II H\&K evolution-SN $2003 \mathrm{~W}$ only has a Ca II H\&K velocity for our $t=-11$ day spectrum, so it is also not included) using $v_{\mathrm{CaH} \& \mathrm{~K}}$ for $\mathrm{SNe}$ Ia with $1 \leqslant \Delta m_{15}(B) \leqslant 1.5 \mathrm{mag}$, we determine the relation

$$
v_{\mathrm{CaH} \& \mathrm{~K}}\left(v_{\mathrm{CaH} \& \mathrm{~K}}^{0}, t\right)=v_{\mathrm{CaH \& K}}^{0}(1-0.0515 t)-0.633 t \text {. }
$$

Examples of this family of functions are shown in Figure 9.

The residuals (as defined in Section 3.1) have a larger scatter $\left(\sigma=570 \mathrm{~km} \mathrm{~s}^{-1}\right)$ than that of Si II $\lambda 6355$, but the residuals are not that large considering other factors contributing to the total uncertainty in this measurement. It is unclear if the larger scatter is the result of a poorer representation of the velocity gradient because of less data, less linear velocity evolution of Ca II H\&K, a less linear relation between velocity at maximum brightness and velocity gradient, interloper measurements, and/or the relation has larger intrinsic scatter. There are also more "catastrophic" outliers (residuals more than $1000 \mathrm{~km} \mathrm{~s}^{-1}$ ), which are likely the remaining absorption velocities that overlap with the blue cloud.

Similar to what was done with Equation (4) and Si II $\lambda 6355$, $v_{\mathrm{CaH \& K}}^{0}$ can be found using Equation (6),

$$
v_{\mathrm{CaH} \& \mathrm{~K}}^{0}=\left(v_{\mathrm{CaH} \& \mathrm{~K}}+0.631 t\right) /(1-0.0513 t) .
$$

Table 1 lists $v_{\mathrm{CaH} \& \mathrm{~K}}^{0}$ for each SN in the F11 sample with a measurement in $-4 \leqslant t \leqslant 12$ days. The value given is for the measurement closest to $t=0$ days.

\section{PSEUDO-EQUIVALENT WIDTH}

Although FK11 showed that ejecta velocity and intrinsic color were connected and we show in Section 5 that the relation is robust, ejecta velocity may not be the best indicator of intrinsic color. FK11 postulated that the redder colors in high-velocity $\mathrm{SNe}$ may be the result of broader lines causing increased line blanketing in the near-UV. If this explanation is correct, one might expect line widths or line strength to be more strongly correlated with intrinsic color.

FWHM and pEW measurements have observational advantages over velocity measurements. Specifically, even if the redshift of a SN Ia is poorly known (e.g., no host galaxy redshift for high- $z \mathrm{SNe}$ ), the FWHM and pEW are still robust. Unfortunately, pEW is sensitive to galaxy light contamination (e.g., Foley et al. 2008a). If FWHM is measured as part of fitting a Gaussian profile to a spectral feature, then deviations from that profile will cause the measured FWHM to lack a physical connection to the data. 


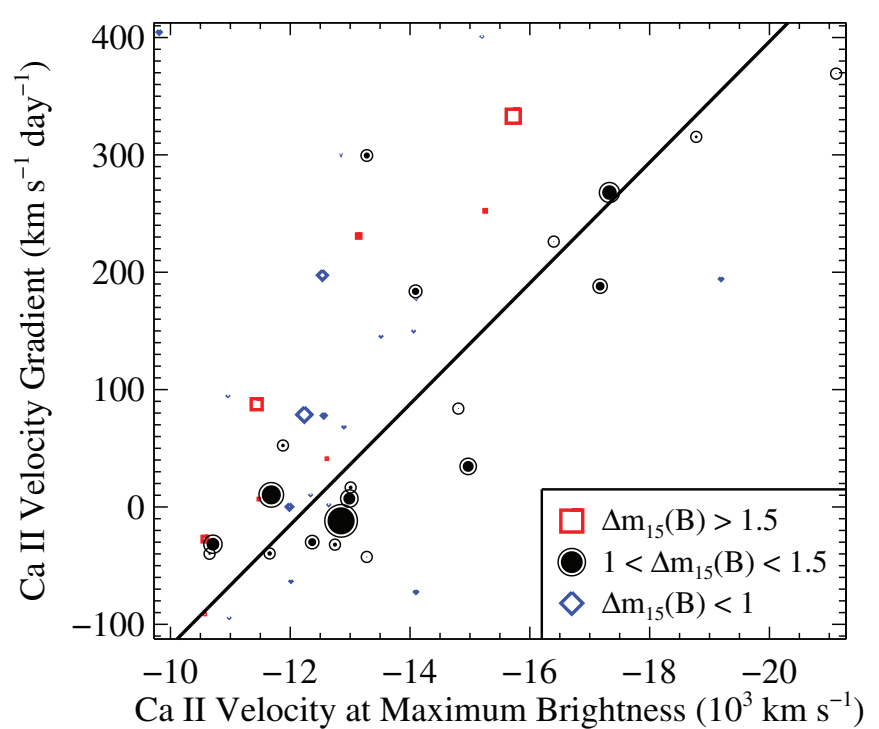

Figure 10. Same as Figure 2, except for Ca II H\&K.

(A color version of this figure is available in the online journal.)

For the F11 sample, the measured Si II $\lambda 6355$ FWHMs assume a Gaussian profile. This has not been attempted for the Ca II H\&K feature since it rarely has a Gaussian profile. We performed the below analysis for the FWHM(Si II) measurements, but found $\mathrm{pEW}(\mathrm{Si}$ II) measurements to yield a more robust result. We therefore only report the pEW results below.

Several groups have examined pEWs of Ca II H\&K and Si II $\lambda 6355$ at both low and high $z$ (e.g., Folatelli 2004; Hachinger et al. 2006; Garavini et al. 2007a; Bronder et al. 2008; Foley et al. 2008a; Balland et al. 2009; Branch et al. 2009; Blondin et al. 2011b; Konishi et al. 2011; Nordin et al. 2011a; Walker et al. 2011). Branch et al. (2006) used the pEW of Si II $\lambda 6355$, along with the pEW of Si II $\lambda 5972$ to subclassify $\mathrm{SNe}$ Ia. His classifications match up well with the Benetti et al. (2005) classifications based on velocity gradient.

\subsection{Si II $\lambda 6355$ Pseudo-equivalent Width}

As noted above, several studies have examined pEWs for several features, including Si II. In Figure 11, we show how $\mathrm{pEW}(\mathrm{Si}$ II $)$ is related to the spectral phase, $\Delta m_{15}(B)$, and $v_{\mathrm{Si} \text { II }}$. There is a wealth of information in these data, but we will only briefly discuss them, leaving further analysis to future work.

Examining Figure 11, we note several observational facts. First, as expected, higher-velocity SNe tend to have stronger lines. Second, slower-declining SNe tend to have weaker lines. Third, lines tend to get stronger over the phase range shown here $(-10<t<10$ days $)$. We also note a generic trend where SNe Ia with $v_{\text {Si II }} \gtrsim-12,000 \mathrm{~km} \mathrm{~s}^{-1}$ can have a large range of $\mathrm{pEW}$ that appears to be completely unrelated to the measured velocity. However, above this velocity, there appears to be a strong relation between $\mathrm{pEW}$ (Si II) and $v_{\mathrm{Si} \text { II }}$ at all phases shown here. This is likely the result of the line saturating. This effect may also be connected to the fact that SNe Ia with $v_{\mathrm{Si} \text { II }}^{0} \gtrsim-12,000 \mathrm{~km} \mathrm{~s}^{-1}$ have a small scatter in intrinsic color, while higher-velocity $\mathrm{SNe}$ Ia have a larger range in intrinsic color (FK11; Section 5).

Figure 12 shows the $\mathrm{pEW}$ evolution as a function of phase for individual SNe. Similar to the method outlined in Section 3, we find a family of functions which describe the $\mathrm{pEW}$ evolution as a function of time. Using the same process as before,

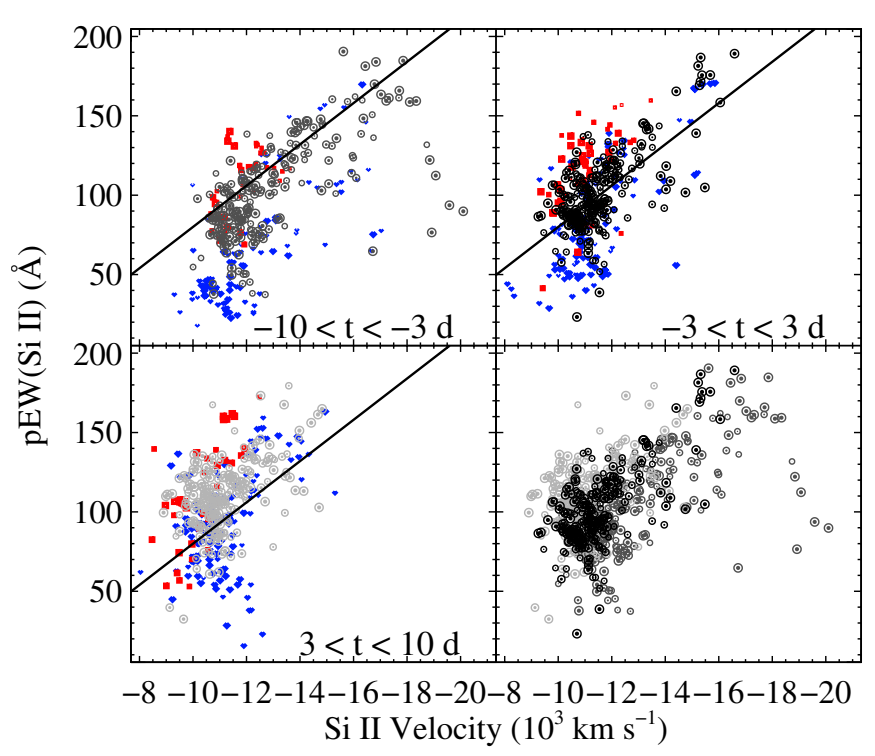

Figure 11. $\mathrm{pEW}\left(\mathrm{Si}\right.$ II) as a function of $v_{\mathrm{Si} \text { II }}$ for different phase and $\Delta m_{15}(B)$ ranges. The panels (clockwise from lower left) show a phase range of $3<t<$ $10,-10<t<-3,-3<t<3$, and $-10<t<10$ days, respectively. The blue diamonds and red squares represent SNe Ia with $\Delta m_{15}(B)<1$ and $\Delta m_{15}(B)>1.5 \mathrm{mag}$, respectively. The dark gray, black, and light gray circles represent SNe Ia with $1<\Delta m_{15}(B)<1.5 \mathrm{mag}$ and $-10<t<-3$, $-3<t<3$, and $3<t<10$ days, respectively. The size of the symbol corresponds to the inverse of the uncertainty. A solid line is plotted to correspond roughly to the relation between $v_{\mathrm{Si} \text { II }}$ and $\mathrm{pEW}(\mathrm{Si}$ II) at early times and high velocity. The same line is plotted in the upper-right and lower-left panels to aid the eye in comparing the different phases. The lower-right panel shows the moderate decliners $\left(1<\Delta m_{15}(B)<1.5 \mathrm{mag}\right)$ for the three phase ranges.

(A color version of this figure is available in the online journal.)

we can determine $\mathrm{pEW}(t=0)=\mathrm{pEW}_{0}$ from a single $\mathrm{pEW}$ measurement in the appropriate phase range $(-7<t<7$ days for $\mathrm{pEW}(\mathrm{Si} \mathrm{II}))$. We find

$$
\mathrm{pEW}_{0}(\mathrm{Si} \text { II })=(\mathrm{pEW}(\mathrm{Si} \mathrm{II})+4.48 t) /(1-0.0339 t) .
$$

In the phase range $-7<t<7$ days, the evolution is relatively linear. For most SNe, the pEW changes $<20 \%$ over this phase range; therefore, knowing the exact evolution of this feature with time is not critical. Unlike $v_{\mathrm{Si} \text { II }}^{0}, \mathrm{pEW}_{0}(\mathrm{Si}$ II $)$ has a relatively Gaussian distribution for our sample.

Table 1 lists $\mathrm{pEW}_{0}(\mathrm{Si}$ II) for each $\mathrm{SN}$ in the F11 sample with a measurement in $-7 \leqslant t \leqslant 7$ days. The value given is for the measurement closest to $t=0$ days.

\subsection{Ca II H\&K Pseudo-equivalent Width}

Unlike $v_{\mathrm{CaH} \& \mathrm{~K}}, \mathrm{pEW}(\mathrm{Ca} \mathrm{II})$ is not systematically affected by multiple absorption components. Although incorrect definition of the borders of the feature can produce incorrect measurements, misidentifying a blue absorption component as a red absorption component does not change any measurements.

Figure 13 shows the $\mathrm{Ca}$ II $\mathrm{H} \& \mathrm{~K}$ pEW evolution as a function of phase for individual SNe. Using the same method outlined in Section 4.1, we fit a family of functions to the near maximum $(-7<t<9$ days) temporal evolution of $\mathrm{pEW}$ (Ca II), which provides a way to convert $\mathrm{pEW}$ measurements in that phase range to a maximum-light measurement, $\mathrm{pEW}_{0}$. We find

$$
\mathrm{pEW}_{0}(\mathrm{Ca} \text { II })=(\mathrm{pEW}(\mathrm{Ca} \text { II })+0.708 t) /(1-0.0210 t) .
$$

Similar to $\mathrm{Si}$ II $\lambda 6355, \mathrm{Ca}$ II $\mathrm{H} \& \mathrm{~K}$ has a relatively Gaussian $\mathrm{pEW}_{0}$ distribution for our sample. 


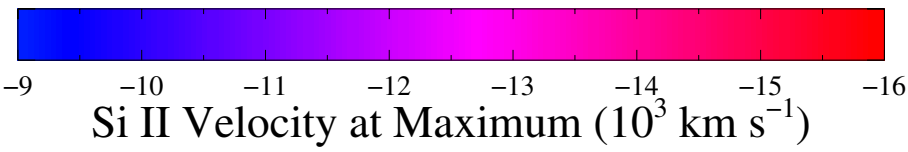

\section{Number \\ $\begin{array}{lllll}5 & 10 & 15 & 20 & 25\end{array}$}

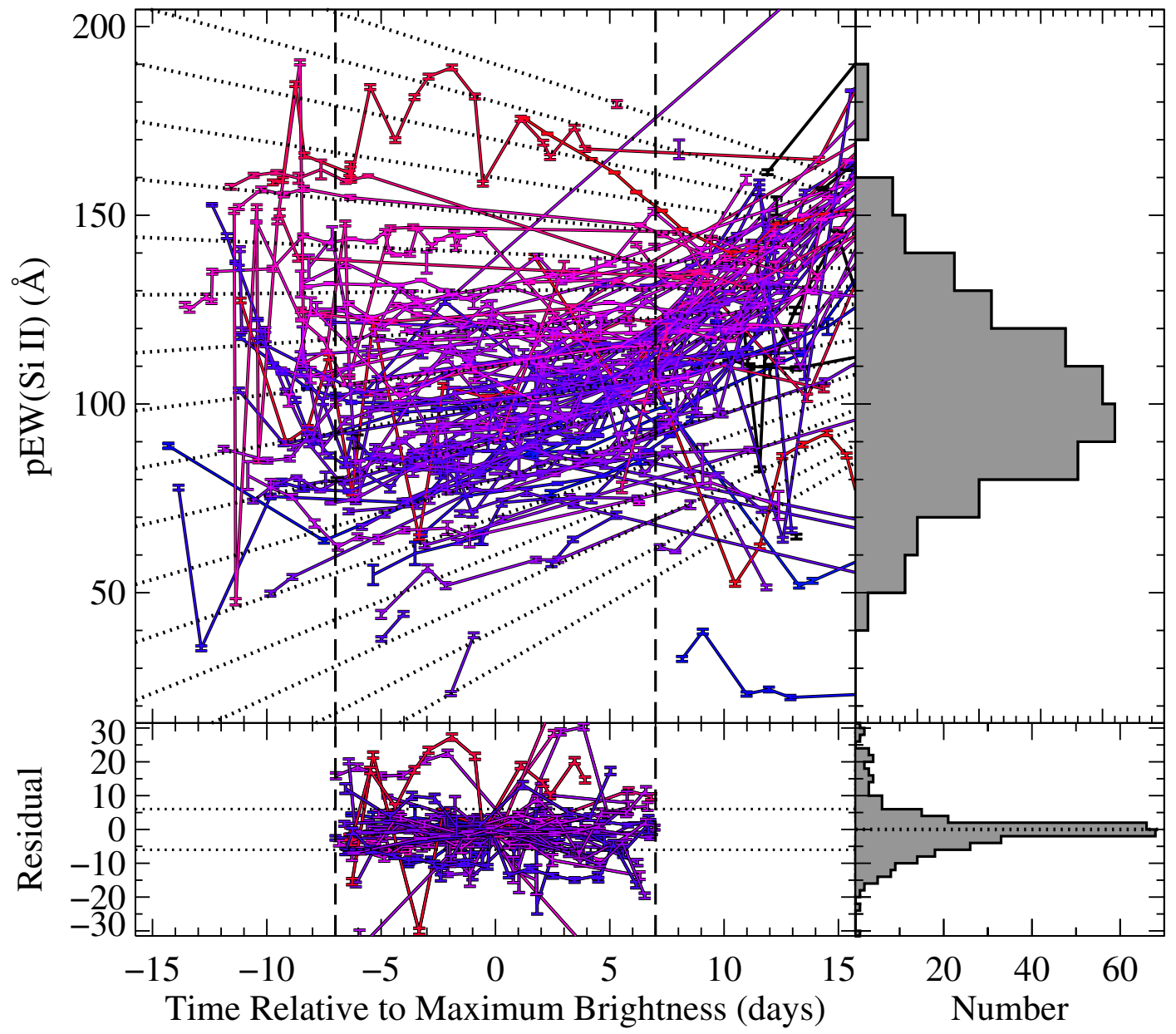

Figure 12. Same as Figure 1, except for pEW(Si II).

(A color version of this figure is available in the online journal.)

Table 1 lists $\mathrm{pEW}_{0}(\mathrm{Ca}$ II) for each $\mathrm{SN}$ in the $\mathrm{F} 11$ sample with a measurement in $-7 \leqslant t \leqslant 9$ days. The value given is for the measurement closest to $t=0$ days.

\section{INTRINSIC COLOR}

\subsection{Matching the F11 Sample to the W09 Sample}

The W09 SN Ia sample is large. It is further useful for various studies since it is separated into two groups by ejecta velocity. However, it lacks published velocity measurements. We are able to provide velocity measurements for 59 of the $121 \mathrm{SNe}$ Ia in the W09 sample (after applying the same cuts as FK11).

W09 used the Si II $\lambda 6355$ velocity evolution of 10 wellobserved low velocity gradient $\mathrm{SNe}$ Ia to determine an average velocity as a function of phase and a dispersion around that average. SNe which had velocity measurements $>3 \sigma$ above the average in the time interval $-7 \leqslant t \leqslant 7$ days were considered "High-Velocity" $\mathrm{SNe}$, while all others with measurements within that time interval were considered "Normal." Since there are intermediate-velocity $\mathrm{SNe}$ and since $\mathrm{SN}$ velocities at later times are similar, this method produces a coarse assessment of the $\mathrm{SNe}$ with potential ambiguity.
Figure 14 shows histograms of $v_{\mathrm{Si} \text { iI }}^{0}$ for the F11 sample of $\mathrm{SNe}$ Ia that overlap with the W09 sample. The two histograms represent $\mathrm{SNe}$ classified as "Normal" and "High-Velocity" by W09. The two subsamples are relatively separate, but there is a significant amount of overlap for $-11,000 \gtrsim v_{\mathrm{Si} \text { II }}^{0} \gtrsim$ $-12,000 \mathrm{~km} \mathrm{~s}^{-1}$. The standard deviation for our relation that measures $v_{\mathrm{Si} \text { II }}^{0}$ is $220 \mathrm{~km} \mathrm{~s}^{-1}$, the typical measurement uncertainty is $\lesssim 100 \mathrm{~km} \mathrm{~s}^{-1}$. (Velocity uncertainty from host-galaxy rotation does not affect this direct comparison.) Given the range of overlap of the subsamples, the data sets and/or methodology must be responsible for some of the overlap. This gives additional motivation for using individual velocity measurements to describe the ejecta velocity of a SN Ia.

\subsection{Intrinsic Color for the W09 Sample}

In Figure 15, we plot the light-curve (but not host-galaxy reddening) corrected peak absolute magnitude of the W09 matched sample as a function of their $B_{\max }-V_{\max }$ pseudocolor. This is similar to Figure 4 from FK11. The symbols representing each $\mathrm{SN}$ are color-coded by $v_{\mathrm{Si} \text { II }}^{0}$. As noted by FK11, the higher-velocity SNe tend to be redder (brighter) for a given peak magnitude (color). 


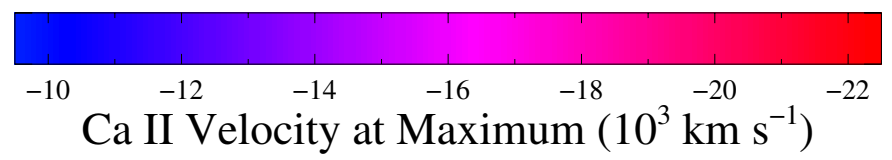

\section{Number}

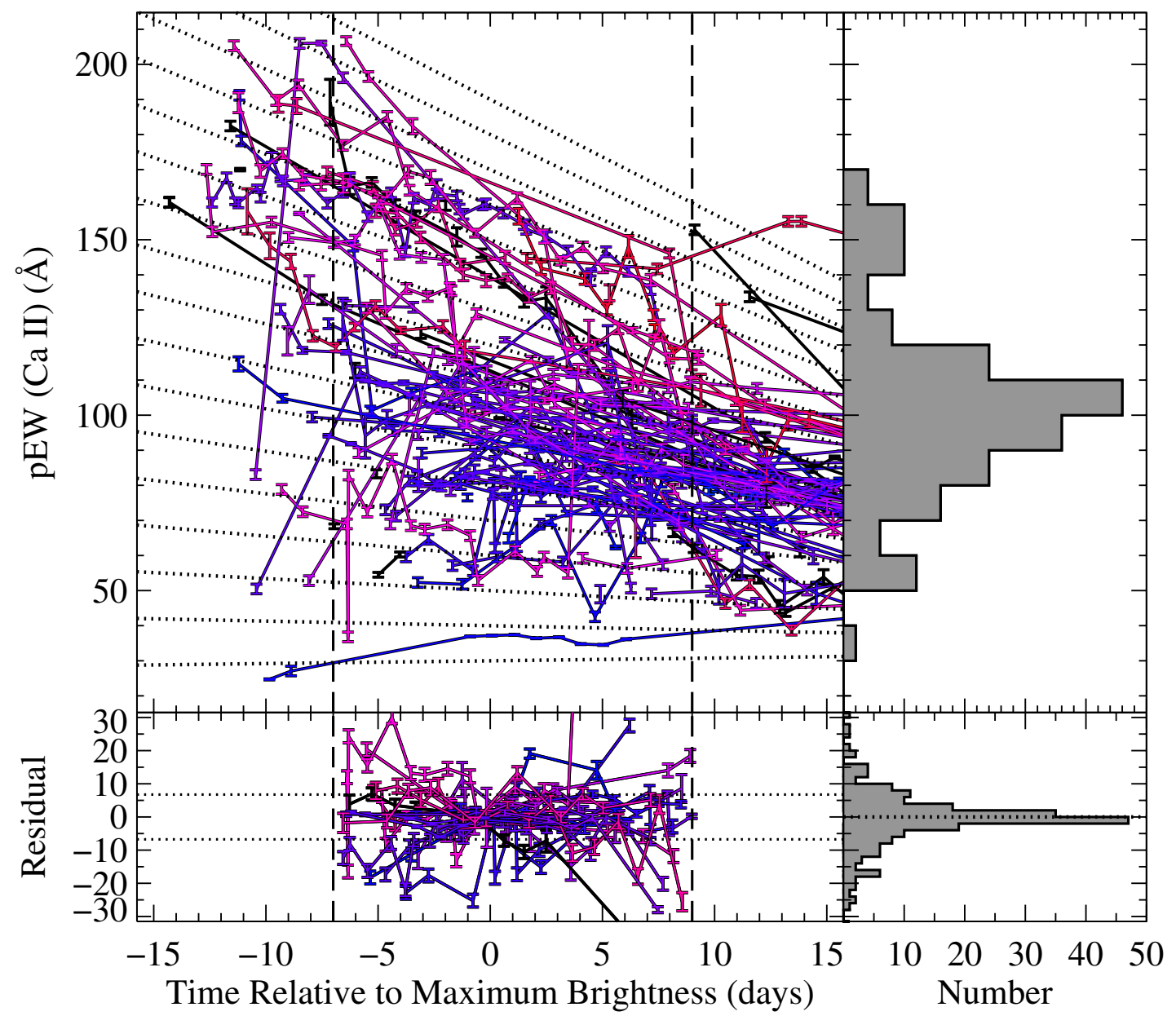

Figure 13. Same as Figure 1, except for pEW(Ca II).

(A color version of this figure is available in the online journal.)

Using Equation (1) from FK11, we have a relation between measured $E(B-V)$ and the peak magnitude. The W09 $E(B-V)$ and the F11 $B_{\max }-V_{\max }$ values are highly correlated. Fitting a line to the data, we find a simple offset of $B_{\max }-V_{\max }=$ $E(B-V)-0.081 \mathrm{mag}$. This is consistent with the offset $(-0.070 \pm 0.012)$ found by Phillips et al. (1999), especially considering the F11 $B_{\max }-V_{\max }$ values include a Milky Way correction. Applying this offset to the best-fit values of Equation (1) of FK11 for the "Normal" SNe, we obtain the solid line in Figure 15. This line represents the dust reddening for a SN Ia with an "intrinsic" color of zero. Although this may not be the true intrinsic color, deviations from this line in the color direction represent deviations from a nominal color that is corrected for host-galaxy reddening. Specifically, we define

$$
\begin{aligned}
\left(B_{\max }-V_{\max }\right)_{0}= & \left(B_{\max }-V_{\max }\right) \\
& -\left(M_{\max , \text { corr }}^{V}-M_{\mathrm{zp}}\right) / R_{V}-C,
\end{aligned}
$$

where $M_{\max , \text { corr }}^{V}=M_{\max }^{V}-\alpha\left(\Delta m_{15}(B)-1.1\right), M_{\mathrm{zp}}=$ $-19.26 \mathrm{mag}, \alpha=0.75, R_{V}=2.5$, and $C=0.081 \mathrm{mag}$ (FK11). This measurement may be offset from the true intrinsic color, but the same offset should apply to all $\mathrm{SNe}$, making the measurement useful for directly comparing $\mathrm{SNe}$.
We restrict the following analysis to $\mathrm{SNe}$ Ia with $B_{\max }-$ $V_{\max }<0.4-0.081=0.319 \mathrm{mag}$, corresponding to the $E(B-V)<0.4 \mathrm{mag}$ cut used by FK11. This is the color range where low- and high-velocity $\mathrm{SNe}$ Ia appear to have the same reddening law (FK11).

Figure 16 presents a histogram of the intrinsic $B_{\max }-V_{\max }$ for the sample. The shape is slightly skewed to red colors, with a skewness of 0.48 . The shape of the color distribution is similar to the color distribution expected from the asymmetric explosion models of Kasen \& Plewa (2007) when accounting for the frequency with which a particular viewing angle is observed.

SN Ia color is correlated with light-curve shape (e.g., Riess et al. 1996; Tripp 1998), with faster declining SNe having redder colors. Maeda et al. (2011) corrected the observed colors of $\mathrm{SNe}$ in their sample using the relation between $B_{\max }-V_{\max }$ and $\Delta m_{15}(B)$ found by Folatelli et al. (2010). Over the full $\Delta m_{15}(B)$ range used for this analysis, the Folatelli et al. (2010) relation predicts a correction of $0.060 \pm 0.025 \mathrm{mag}$. Although there is only a slight correlation between light-curve shape and ejecta velocity for the range of $\Delta m_{15}(B)$ examined here (e.g., Figure 3 ), there may still be a significant correlation between $\Delta m_{15}(B)$ and intrinsic color for the W09-F11 matched sample. 


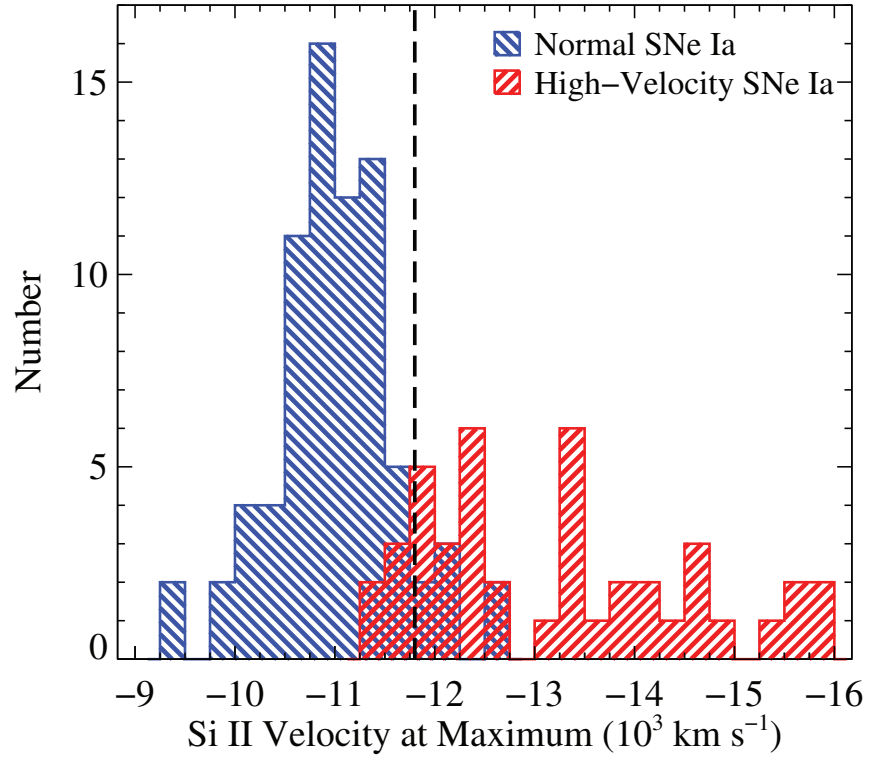

Figure 14. Histograms of $v_{\mathrm{SiII}}^{0}$ measured from the F11 spectra. The blue and red histograms represent SNe classified as "Normal" and "High-Velocity," respectively, by W09. The dashed line represents the value of $v_{\mathrm{S} \text { II }}^{0}$ that W09 nominally used to separate the two subsamples.

(A color version of this figure is available in the online journal.)

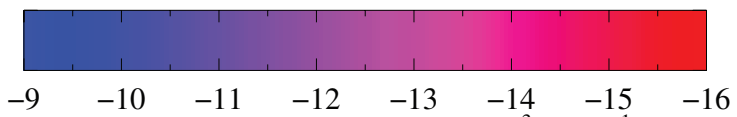
Si II Velocity at Maximum $\left(10^{3} \mathrm{~km} \mathrm{~s}^{-1}\right)$

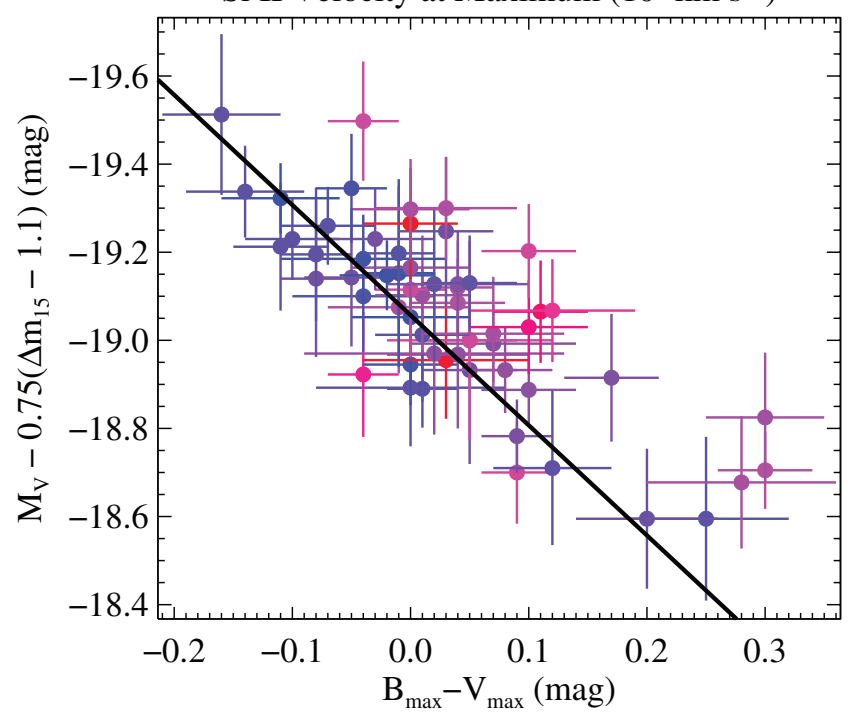

Figure 15. Light-curve shape corrected peak absolute $V$ brightness as a function of $B_{\max }-V_{\max }$. The color of the symbol corresponds to the SN's $v_{\mathrm{SiI}}^{0}$, with the color bar at the top of the figure displaying the correspondence. The solid black line represents the host-galaxy dust reddening of a zero-color SN Ia. Deviations from this line in color are the intrinsic color of the SN.

(A color version of this figure is available in the online journal.)

Figure 17 shows the distribution of color and light-curve shape for the W09-F11 matched sample. The two quantities displayed $\left(B_{\max }-V_{\max }\right.$ and $\left.\Delta m_{15}(B)\right)$ are not correlated for the sample, having a correlation coefficient of 0.04 . Therefore, the intrinsic color is not significantly affected by light-curve shape for the sample. Given the uncertainty in the Folatelli et al. (2010) relation, the fact that it was derived using a combination of low- and high-velocity SNe Ia, the small correction expected

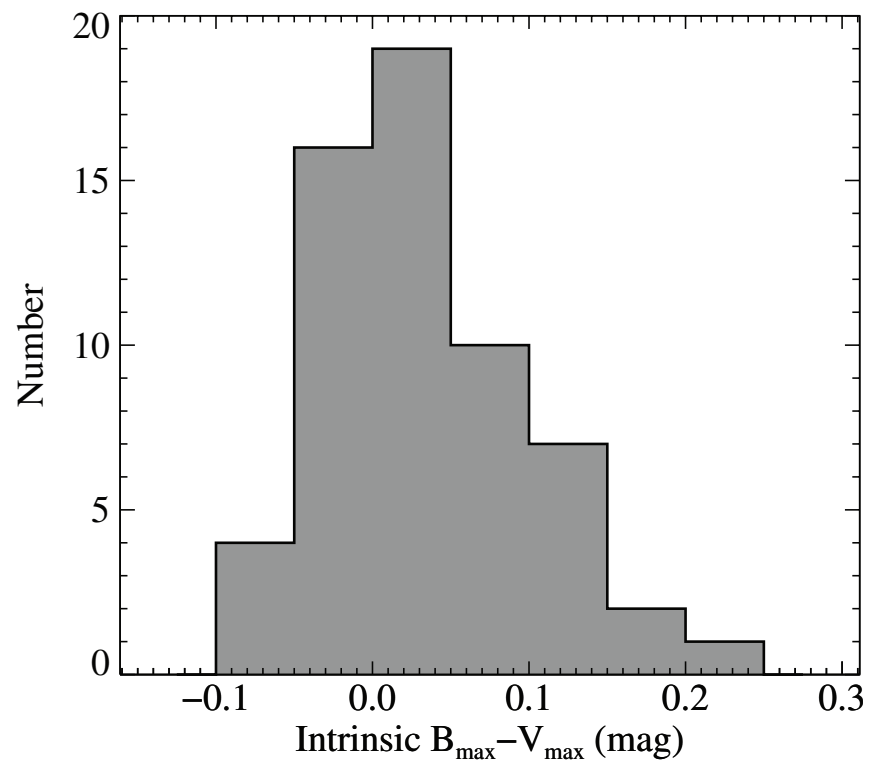

Figure 16. Distribution of intrinsic $B_{\max }-V_{\max }$ for the W09-F11 matched sample of SNe Ia.

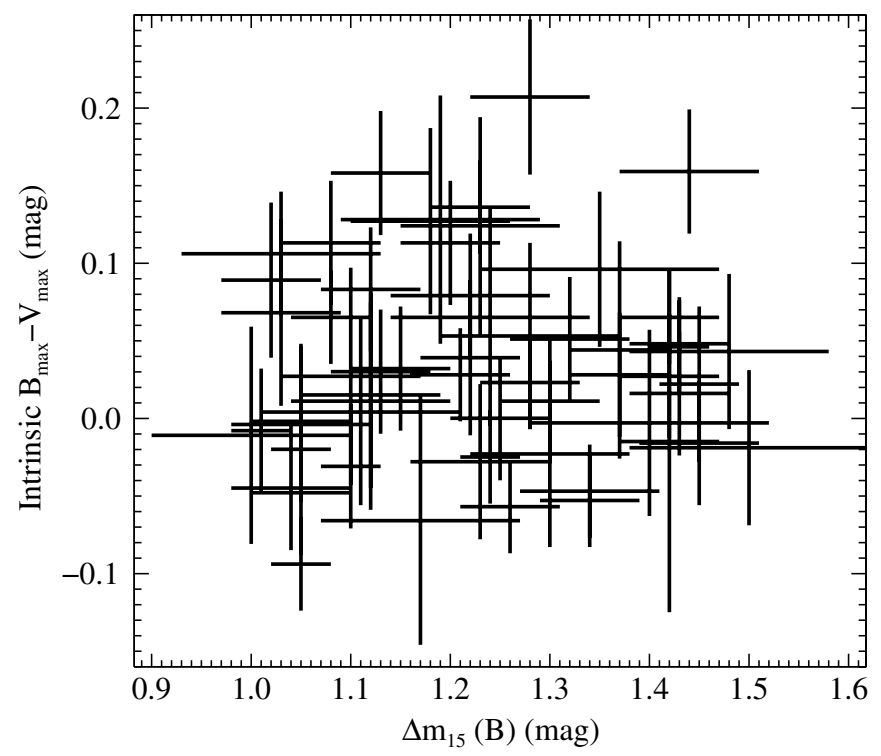

Figure 17. Intrinsic $B_{\max }-V_{\max }$ pseudo-color as a function of $\Delta m_{15}(B)$ for the W09-F11 sample. The correlation coefficient is 0.04 .

for SNe Ia in this analysis, and the lack of a correlation between light-curve shape and intrinsic color for the sample, we do not apply this correction.

In Figure 18, we compare the intrinsic color of each SN to its $v_{\mathrm{Si}}^{0}$. There is a trend where $\mathrm{SNe}$ with redder intrinsic colors systematically have higher ejecta velocity. The correlation between the two quantities is 0.28 , and a linear least-squares fit results in a slope that is significant at the $3.4 \sigma$ level. Performing a Bayesian Monte-Carlo linear regression on the data (Kelly 2007), we find that $99.142 \%$ of the realizations have a negative slope. The trend is more apparent when separating the sample into equal-numbered groups based on $v_{\mathrm{Si} \text { II }}^{0}$ and examining their median values. The trend is also qualitatively similar to that found by FK11 for the synthetic spectra of Kasen \& Plewa (2007).

From the W09 sample, it is unclear if the trend is linear or if there is a simple color offset. There is a larger intrinsic color scatter for the higher-velocity $\mathrm{SNe}$ than for the 


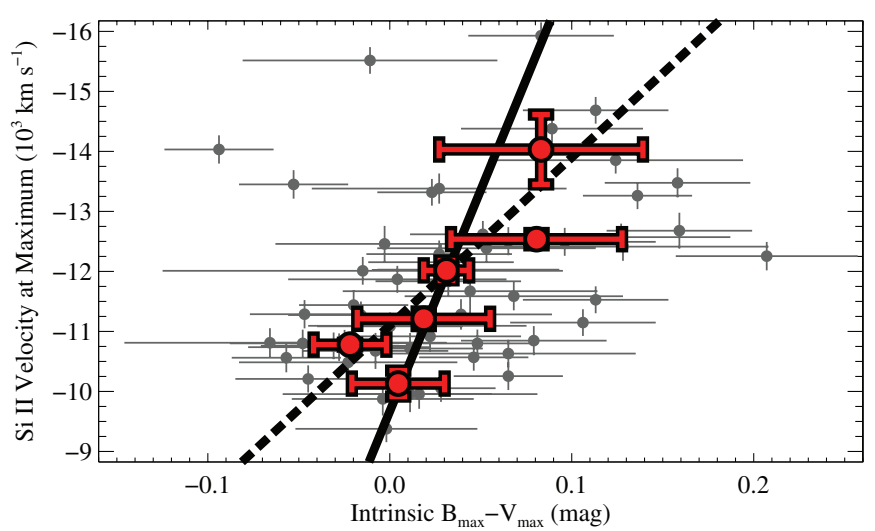

Figure 18. Maximum-light $\mathrm{Si}$ II $\lambda 6355$ velocity $\left(v_{\mathrm{Si} \text { II }}^{0}\right)$ as a function of intrinsic $B_{\max }-V_{\max }$ pseudo-color for the W09 sample of SNe Ia. The red circles represent the median values of equal-numbered velocity bins with the error bars being the median absolute deviation. The dashed line is the relation between velocity and color for the Kasen \& Plewa (2007) models (Equation (3) of FK11). The solid line is the best-fit linear model for the data.

(A color version of this figure is available in the online journal.)

lower-velocity SNe. Splitting the sample by $v_{\text {Si II }}^{0}=$ $-11,800 \mathrm{~km} \mathrm{~s}^{-1}$, the intrinsic color scatter is 0.070 and 0.047 mag for the high- and low-velocity subsamples, respectively. This was postulated by FK11 as the reason that the "Normal" SNe Ia produced a smaller scatter in Hubble residuals than the "High-Velocity" SNe Ia. However, even after removing a linear trend between velocity and intrinsic color, the highervelocity $\mathrm{SNe}$ Ia continue to have a higher intrinsic color scatter (0.071 versus $0.046 \mathrm{mag}$ ). This suggests that $\mathrm{SNe}$ Ia with lower ejecta velocity may be intrinsically better distance indicators and with enough $\mathrm{SNe}$ Ia, it may be prudent to reject high-velocity $\mathrm{SNe}$ Ia from cosmological samples.

\subsection{Intrinsic Color for the F11 Sample}

As described in Section 2.1, there are some drawbacks to using the W09 data. Here we repeat the above analysis to determine the intrinsic color of SNe Ia, but use the F11 sample. Specifically, derived photometric values have been compiled from CfA3 (Hicken et al. 2009a) and LOSS (Ganeshalingam et al. 2010). The CfA3 peak magnitudes were corrected to match the LOSS system. See Section 2.1 for details. We derive intrinsic $B_{\max }-V_{\max }$ pseudo-colors for each SN in the F11 sample. Using the measurements of $v_{\mathrm{Si} \text { II }}, v_{\mathrm{CaH} \& \mathrm{~K}}, \mathrm{pEW}(\mathrm{Si}$ II), and $\mathrm{pEW}(\mathrm{Ca}$ II $)$ from $\mathrm{CfA}$ and literature spectra near maximum brightness and the time evolution of these quantities as defined by Equations (5), (7), (8), and (9), we derive $v_{\mathrm{Si} \text { II }}^{0}, v_{\mathrm{CaH} \& \mathrm{~K}}^{0}$, $\mathrm{pEW}_{0}(\mathrm{Si} \mathrm{II})$, and $\mathrm{pEW} \mathrm{W}_{0}(\mathrm{Ca} \mathrm{II})$, respectively, for individual SNe. Below, we will compare these values to their intrinsic colors. The F11 sample takes no data directly from W09, but the samples have some $\mathrm{SNe}$ in common and may share derived light-curve values. Furthermore, the $v_{\mathrm{Si} \text { II }}^{0}$ values used in the above analysis are identical to those presented here for the SNe in both samples.

Similar to what was done in Section 5.2, we restrict the following analysis to SNe Ia with $B_{\max }-V_{\max }<0.319 \mathrm{mag}$, the color range where low- and high-velocity SNe Ia appear to have the same reddening law (FK11). We exclude SNe Ia with $z<0.01$, except for those with Cepheid distances from Riess et al. (2011). There are 65, 59, 42, and $49 \mathrm{SNe}$ Ia with measured maximum-brightness light-curve parameters, $1 \leqslant \Delta m_{15}(B) \leqslant$ $1.5 \mathrm{mag}$, and a maximum-brightness spectral parameter for $v_{\mathrm{Si} \text { II }}^{0}$, $\mathrm{pEW}_{0}(\mathrm{Si}$ II $), v_{\mathrm{CaH} \& \mathrm{~K}}^{0}$, and $\mathrm{pEW}_{0}(\mathrm{Ca} \mathrm{II})$, respectively.

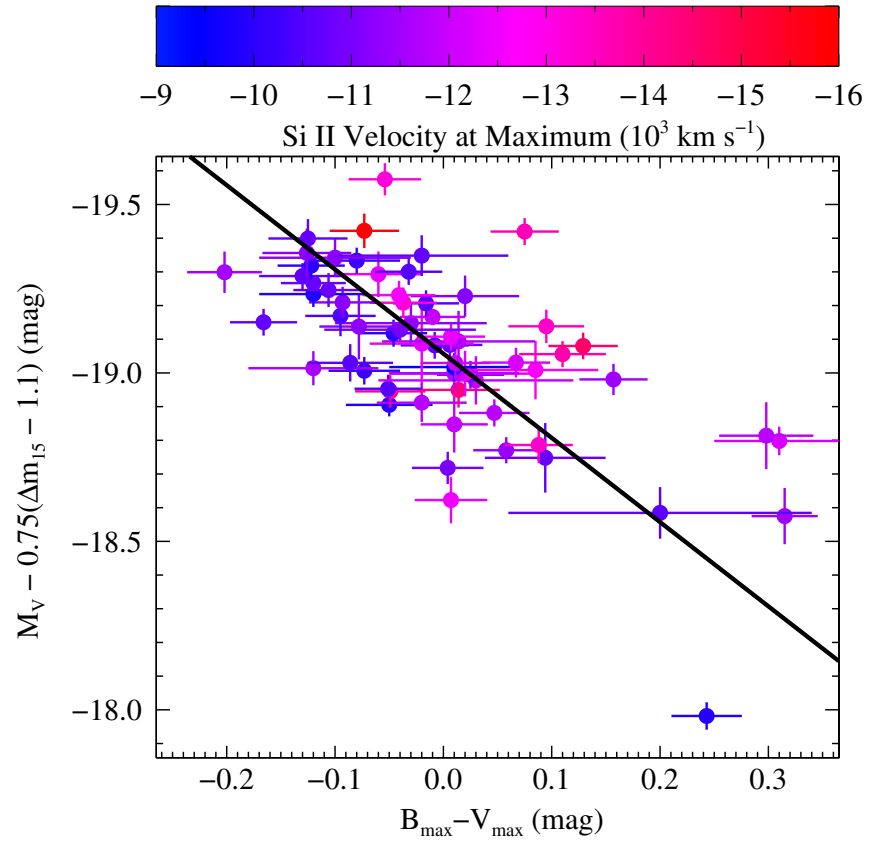

Figure 19. Same as Figure 15, except for the F11 sample. (A color version of this figure is available in the online journal.)

Using the $v_{\mathrm{Si} \text { II }}^{0}$ data, Figure 19 shows the light-curve (but not host-galaxy reddening) corrected peak absolute magnitude of the F11 sample as a function of their $B_{\max }-V_{\max }$ pseudo-color. This is similar to Figure 15.

As was seen in the W09 sample, the higher-velocity SNe Ia in the F11 sample tend to be redder than lower-velocity SNe Ia. Using the procedure outlined above, we again calculate the intrinsic color for the sample. Figure 20 compares the intrinsic color of each SN to its ejecta velocity (as derived from Si II $\lambda 6355$ and $\mathrm{Ca}$ II $\mathrm{H} \& \mathrm{~K}$ ) and $\mathrm{pEW}$ values.

As with the W09 sample, the F11 sample shows a strong trend between $v_{\mathrm{Si} \text { II }}^{0}$ and intrinsic color such that intrinsically redder SNe Ia tend to have higher velocity ejecta. Unlike the W09 sample, the F11 sample has an obvious linear relation between these quantities - not just an offset, corresponding to

$$
\begin{aligned}
\left(B_{\max }-V_{\max }\right)_{0} & =(-0.39 \pm 0.04)-(0.033 \pm 0.004) \\
& \times\left(v_{\mathrm{Si}_{\text {II }}}^{0} / 1000 \mathrm{~km} \mathrm{~s}^{-1}\right) \mathrm{mag} .
\end{aligned}
$$

Performing a linear least-squares fit results in a non-zero slope that is significant at the $8.8 \sigma$ level. The correlation between the two quantities is -0.39 . Performing a Bayesian analysis of Monte-Carlo linear regressions on the data (Kelly 2007), we find that $99.949 \%$ of the realizations have a negative slope and the median correlation coefficient for the realizations is -0.47 . There is a reasonable amount of scatter to the relation, but on average, the trend is quite clear. Although slightly offset, the linear relation is strikingly similar to the theoretical trend found by FK11.

Similar to what was found with the W09 sample, the intrinsic color scatter is larger for higher-velocity (as determined by $\left.v_{\mathrm{Si} \text { II }}^{0}\right) \mathrm{SNe}$ Ia. The intrinsic color scatter for SNe Ia with $v_{\mathrm{Si} \text { II }}^{0}$ higher/lower than $-11,800 \mathrm{~km} \mathrm{~s}^{-1}$ is 0.095 and $0.072 \mathrm{mag}$, respectively. After correcting for the linear trend, the scatter does not change much, resulting in a scatter of 0.093 and 0.070 mag for the subsamples, respectively. However, correcting for the linear trend reduces the overall scatter from $\sigma=0.087$ to $0.080 \mathrm{mag}$. 


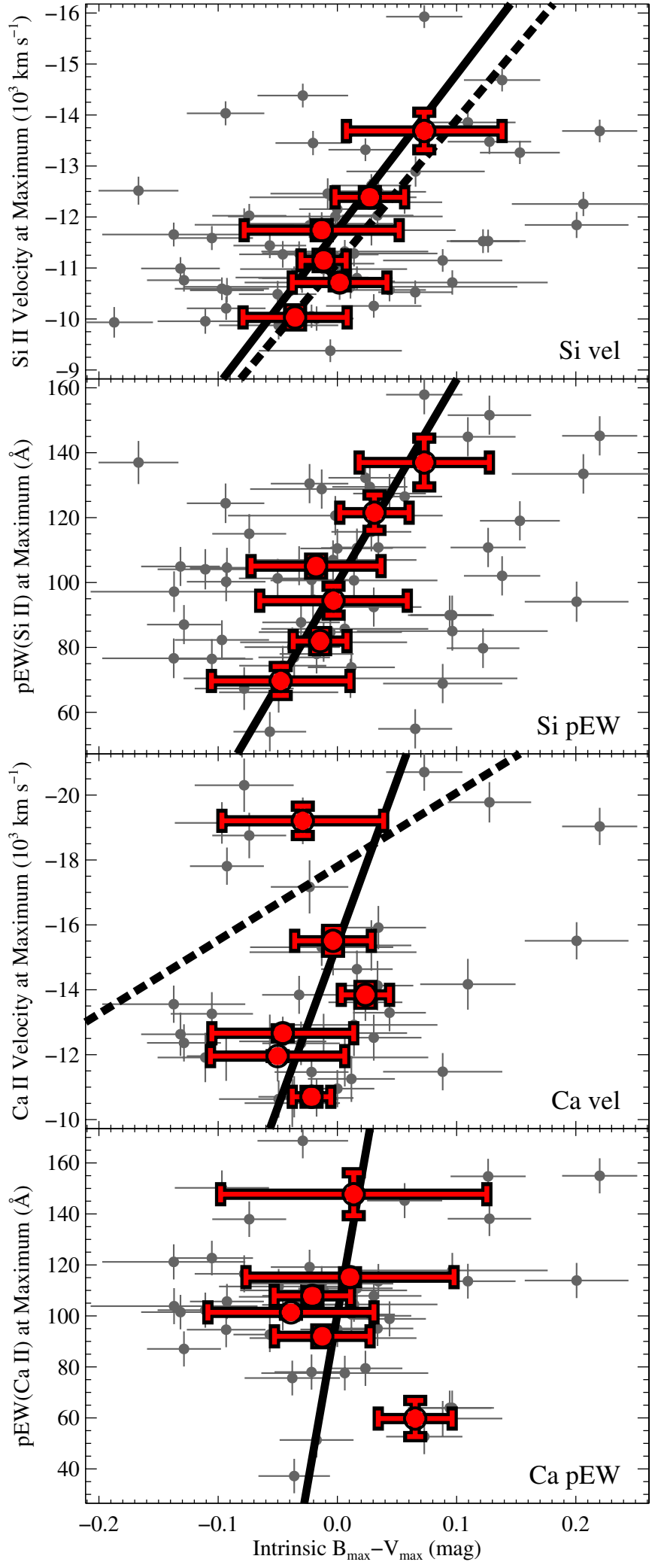

Figure 20. Maximum-light Si II $\lambda 6355$ velocity, pEW(Si II), Ca II H\&K velocity, and $\mathrm{pEW}\left(\mathrm{Ca}\right.$ II) as a function of intrinsic $B_{\max }-V_{\max }$ pseudo-color (top to bottom panel, respectively) for the F11 sample of SNe Ia. The top panel is similar to Figure 18, except for the F11 sample. The red circles represent the median values of equal-numbered velocity or $\mathrm{pEW}$ bins with the error bars representing the median absolute deviation. The dashed lines in the top and third panels are the relation between velocity and color for the Kasen \& Plewa (2007) models (Equations (3) and (4) of FK11, respectively). The theoretical relations are strikingly similar and particularly poor for the Si II $\lambda 6355$ and Ca II $\mathrm{H} \& \mathrm{~K}$ data, respectively. The solid lines are the best-fit linear model for the data. (A color version of this figure is available in the online journal.)
Comparing $\mathrm{pEW}_{0}\left(\mathrm{Si}\right.$ II) to the intrinsic $B_{\max }-V_{\max }$ pseudocolor (Figure 20), there is a reasonable correlation $(\rho=0.28)$, with a linear relation defined by

$$
\begin{aligned}
\left(B_{\max }-V_{\max }\right)_{0}= & (-0.16 \pm 0.03)-(0.0016 \pm 0.0003) \\
& \times \mathrm{pEW}_{0}(\mathrm{Si} \text { II }) \mathrm{mag} .
\end{aligned}
$$

The slope of the best-fit line for the two quantities is $6.3 \sigma$ from zero. Again, looking at medians (now for bins of equal number in pEW space), the trend is clear. Performing a Bayesian analysis of Monte-Carlo linear regressions on the data (Kelly 2007), we find that $98.6 \%$ of the realizations have a positive slope and a median correlation coefficient of 0.34 . The intrinsic color of SNe Ia can be derived from both the velocity and $\mathrm{pEW}$ of Si II $\lambda 6355$ near maximum brightness.

The CaII H\&K measurements produce less robust results. Intrinsic color is correlated with $v_{\mathrm{CaH} \& \mathrm{~K}}^{0}$, where (similar to Si II 26355$)$ SNe Ia with higher velocity ejecta tend to be intrinsically redder. A linear least-squares fit to the data results in a slope that is $4.7 \sigma$ from zero. However, the data have a large scatter and the correlation is not apparent by eye $(\rho=-0.24)$. A Bayesian Monte-Carlo analysis of the data results in $96.1 \%$ of the realizations having a negative slope and a median correlation coefficient of -0.33 . Nonetheless, lower-velocity SNe Ia, as determined from Ca II H\&K, appear to have less intrinsic color scatter than higher-velocity SNe Ia. Splitting the sample at $v_{\mathrm{CaH} \& \mathrm{~K}}^{0}=-14,000 \mathrm{~km} \mathrm{~s}^{-1}$, we find that the higher/lower-velocity SNe Ia have intrinsic color scatter of 0.095 and $0.058 \mathrm{mag}$, respectively. Therefore, a velocity cut may improve cosmological results. The linear fit to the data is significantly different from that found by FK11 for the Kasen \& Plewa (2007) models, and detrending the data by the best-fit line slightly increases $(\sim 0.003 \mathrm{mag})$ the scatter of the velocity subsamples (but decreases the scatter of the full sample by $\sim 0.002 \mathrm{mag}$ ).

The $\mathrm{pEW}$ (Ca II) measurements do not show a correlation with intrinsic color. The correlation coefficient is 0.03 , and there is no significant linear trend as found by a fit to the data (performing the similar linear fits provided a non-zero slope at the $1.8 \sigma$ level and positive slopes for $65.1 \%$ of the realizations). There is no clear way to make a cut on $\mathrm{pEW}(\mathrm{Ca}$ II) to reduce the intrinsic color scatter. Two competing effects drive the value of pEW(Ca II). First, higher velocity ejecta correspond to broader lines (as clearly seen for Si II $\lambda 6355$; Figure 11 ), so SNe Ia with higher ejecta velocity, which have redder intrinsic colors, should have higher $\mathrm{pEW}$. However, $\mathrm{SNe}$ Ia with redder intrinsic $B-V$ colors, which have higher ejecta velocity, will have a depressed UV continuum, and should have a lower pEW. These competing effects may reduce any correlation for $\mathrm{pEW}$ (Ca II).

\section{DISCUSSION AND CONCLUSIONS}

SN Ia kinematics (as measured by the minimum of the absorption or the width of the absorption of spectral features) are related to the intrinsic color of a $\mathrm{SN}$ Ia. The correlation between SN Ia ejecta velocity and intrinsic color found by FK11 has been further investigated here by examining additional kinematic probes: velocity and $\mathrm{pEW}$ measurements for both $\mathrm{Si}$ II $\lambda 6355$ and $\mathrm{Ca}$ II H\&K. Using spectral series of many SNe Ia, families of functions, which provide a measurement of velocity and $\mathrm{pEW}$ at maximum brightness given a measurement near maximum brightness, were constructed. Both features show a strong correlation between their velocity gradient and velocity at maximum brightness such that $\mathrm{SNe}$ Ia with larger velocity 


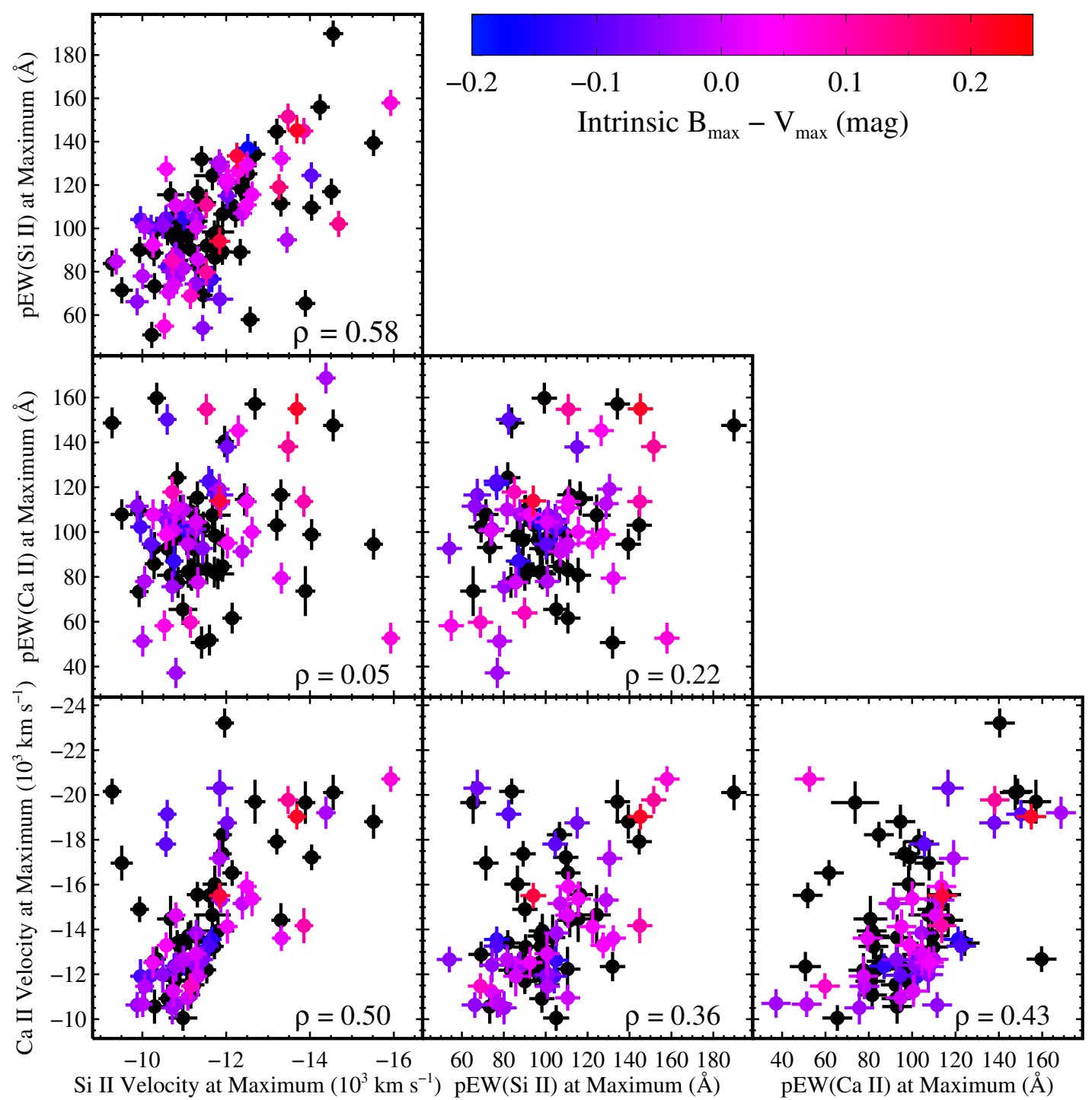

Figure 21. Maximum-light Si II $\lambda 6355$ velocity, pEW(Si II), Ca II H\&K velocity, and pEW(Ca II) for the F11 sample. SNe with no intrinsic color information are black, while those with intrinsic color information are color-coded by their intrinsic color with the mapping represented by the color bar. The Pearson correlation coefficient for each set of parameters is noted in each panel.

(A color version of this figure is available in the online journal.)

gradients tend to have higher velocities at maximum brightness. Similar to high velocity gradient SNe (Hachinger et al. 2006), higher-velocity SNe also tend to have broader features (see also W09). Restricting the sample to $1 \leqslant \Delta m_{15}(B) \leqslant 1.5 \mathrm{mag}$, we find no correlation between host-galaxy morphology and $v_{\mathrm{Si} \text { iा }}^{0}$. Using the families of functions, SNe Ia with spectra at different phases can be directly compared. These functions provide a way to directly compare $\mathrm{SNe}$ Ia using a single near maximum spectrum, providing an easy way to categorize $\mathrm{SNe}$ Ia in a manner similar to how $\Delta m_{15}$ has been used for photometry. Using the photometric parameters of W09 as well as Hicken et al. (2009a) and Ganeshalingam et al. (2010) along with the reddening law derived in FK11, we are able to determine the intrinsic color of both the W09 and F11 samples, respectively.

Comparing the maximum-light velocity and $\mathrm{pEW}$ measurements with the intrinsic color data for individual $\mathrm{SNe}$, we find correlations between intrinsic color and $v_{\mathrm{Si} \text { II }}^{0}, v_{\mathrm{CaH} \& \mathrm{~K}}^{0}$, and $\mathrm{pEW}_{0}\left(\mathrm{Si}\right.$ II), although the correlation with $v_{\mathrm{CaH} \& \mathrm{~K}}^{0}$ is less robust. We find no correlation between intrinsic color and $\mathrm{pEW}_{0}(\mathrm{Ca}$ II $)$, and speculate that the competing effects of higher-velocity $\mathrm{SNe}$
Ia having broader lines and depressed UV continua balance each other to some extent, negating any correlation. The intrinsic color of a SN Ia varies linearly with both $v_{\mathrm{Si} \text { II }}^{0}$ and $\mathrm{pEW}_{0}(\mathrm{Si}$ II), although there is significant scatter.

Thus far, we have only discussed certain aspects of how our measured parameters correlate with each other. Specifically, we find that $v_{\mathrm{Si} \text { II }}$ and $v_{\mathrm{CaH} \& \mathrm{~K}}$ are highly correlated (Figure 8). We also find that $v_{\mathrm{Si} \text { II }}$ and $\mathrm{pEW}$ (Si II) are highly correlated for the highest velocities, but uncorrelated at lower velocities (Figure 11). In Figure 21, $v_{\mathrm{Si} \text { II }}^{0}, \mathrm{pEW}_{0}(\mathrm{Si} \mathrm{II}), v_{\mathrm{CaH} \& \mathrm{~K}}^{0}$, and $\mathrm{pEW}_{0}(\mathrm{Ca}$ II) are plotted against each other for the F11 sample. $\mathrm{SNe}$ with intrinsic color information are color-coded by their intrinsic color. Most parameters are highly correlated with each other (the absolute Pearson correlation coefficient for each combination is presented in Figure 21). The exceptions are $v_{\mathrm{Si} \text { II }}^{0}$ and $\mathrm{pEW}_{0}\left(\mathrm{Ca}\right.$ II), and $\mathrm{pEW}_{0}$ (Si II) and $\mathrm{pEW}_{0}$ (Ca II). This is not unexpected since $v_{\mathrm{S}_{\text {II }}}^{0}$ and $\mathrm{pEW}_{0}(\mathrm{Si}$ II $)$ correlate with intrinsic color while $\mathrm{pEW}_{0}(\mathrm{Ca}$ II) does not.

There are a handful of outliers for each relation between the spectral parameters, but the outliers do not appear to have 
a strong relation with intrinsic color. Therefore, measuring multiple parameters may not directly improve our knowledge of intrinsic color. Moreover, it is not clear if rejecting SNe based on their placement in this multidimensional parameter space would improve any of the previously determined relations.

Explosion models combined with radiative transfer models must account for the relation between ejecta kinematics and intrinsic color. Accurate SN Ia models should reproduce both the general trends seen in our data as well as the scatter. We find that high-velocity (as defined by either $v_{\mathrm{Si} \text { II }}^{0}$ or $v_{\mathrm{CaH} \& \mathrm{~K}}^{0}$ ) $\mathrm{SNe}$ Ia have a larger scatter in their intrinsic color even after accounting for the velocity-color relation. FK11 suggested that the larger Hubble scatter seen for "High-Velocity" SNe Ia is caused primarily by the larger scatter in intrinsic color, but also suggested that this is primarily driven by the larger velocity range for those $\mathrm{SNe}$. This appears to be correct, but the residual scatter after correcting for velocity indicates lower-velocity SNe Ia are better standard crayons than higher-velocity SNe Ia.

To reduce the scatter and potential bias (FK11) of their samples, future cosmological analyses with SNe Ia may elect to reject higher-velocity $\mathrm{SNe}$ Ia, along with or instead of fitting a relation between velocity and color. Furthermore, the most precise and accurate SN Ia distances must account for this effect. FK11 outlined how ignoring this effect will increase the scatter associated with SN Ia distances and may bias those distances, resulting in biased measurements of cosmological parameters.

Although we provide a prescription for determining the intrinsic color of SNe Ia with a rest-frame optical spectrum near maximum brightness, this relation is not robust for highvelocity SNe Ia if the spectrum does not cover Si II $\lambda 6355$. For $\mathrm{SNe}$ Ia at $z \gtrsim 0.4$, this feature is rarely observed. Alternatively, a relatively homogeneous data set can be obtained by making a cut based on $\mathrm{Ca}$ II $\mathrm{H} \& \mathrm{~K}$ velocity. $\mathrm{SNe}$ Ia with $v_{\mathrm{CaH} \& \mathrm{~K}}^{0} \gtrsim-14,000 \mathrm{~km} \mathrm{~s}^{-1}$ have a relatively small range of intrinsic $B_{\max }-V_{\max }$ pseudo-color $(\sigma=0.06 \mathrm{mag})$. To avoid biases and to produce the most precise and accurate SN Ia distances, all future SN Ia cosmology surveys should strongly consider obtaining spectroscopy near maximum light for their primary cosmological sample. Measuring Si II $\lambda 6355$ appears to have some advantages over the easier to observe Ca II H\&K. Therefore, surveys targeting $\mathrm{SNe}$ Ia at $z>0.4$ may want to explore NIR spectroscopy. Even Ca II H\&K is redshifted out of the optical window for $z \gtrsim 1.2$, making NIR spectroscopy necessary for the highest-redshift SNe Ia. We note that if one has a detector that is sensitive to $2 \mu \mathrm{m}$, one can in principle measure Si II $\lambda 6355$ and $\mathrm{Ca}$ II $\mathrm{H} \& \mathrm{~K}$ to $z \approx 2.2$ and $z \approx 4$, respectively.

We could not have performed the analysis presented here without the large CfA data set. Although further analysis with an expanded sample is necessary to strengthen the claims presented here and examine the potential for combining different measurements to improve our knowledge of the intrinsic color, the current sample has provided insight and direction for these future studies. To expand the current sample, our group will soon release its CfA4 sample of SN Ia light curves. On a similar timescale, the Carnegie Supernova Project (CSP) will provide its next sample of light curves. For spectroscopy, the Berkeley Supernova Ia Program (BSNIP) will soon release its sample of SN Ia spectra, including all spectra used in the W09 analysis. Similarly, the CSP will soon release their spectroscopic data set. While the BSNIP sample will not have the well-sampled spectral series of the CfA and CSP samples, it does have spectra of many SNe Ia near maximum brightness, and its spectra typically cover bluer and redder wavelengths than the CfA spectra. Therefore, it should provide many additional $\mathrm{SNe}$ for this particular type of analysis. On a slightly longer timescale, the Palomar Transient Factory (Rau et al. 2009) and Supernova Factory (Aldering et al. 2002) will provide light curves and spectra of thousands of $\mathrm{SNe}$ Ia. With these combined data sets, it should be possible to further refine and extend the results presented here.

R.J.F. is supported by a Clay Fellowship. N.E.S. is supported by the National Science Foundation through a Graduate Research Fellowship. Supernova studies at the Harvard College Observatory are supported by NSF grant AST09-07903.

This work would not have been possible without the tireless work of S. Blondin, who reduced the CfA spectra and made the spectral measurements. His comments have also significantly improved this work. P. Berlind and M. Calkins spent countless nights observing at the $1.5 \mathrm{~m}$ telescope and obtained most of the spectra used for this study. We thank them for their dedication. We thank many observers who contributed spectra to our Literature sample and especially thank those who provided velocity measurements in the circulars and telegrams. R.J.F. thanks S. Jha and K. Mandel, who influenced the direction of this work. M. Hicken and M. Ganeshalingam graciously contributed information regarding the CfA3 and LOSS photometry necessary for this study. Some ideas presented here were first discussed at the Aspen Center for Physics during the Summer 2010 workshop, "Taking Supernova Cosmology into the Next Decade."

\section{REFERENCES}

Aldering, G., Adam, G., Antilogus, P., et al. 2002, Proc. SPIE, 4836, 61 Aldering, G., \& Conley, A. 2000, IAU Circ., 7413, 2

Altavilla, G., Stehle, M., Ruiz-Lapuente, P., et al. 2007, A\&A, 475, 585 Amanullah, R., Lidman, C., Rubin, D., et al. 2010, ApJ, 716, 712 Anupama, G. C., Sahu, D. K., \& Jose, J. 2005, A\&A, 429, 667 Bailey, S., Aldering, G., Antilogus, P., et al. 2009, A\&A, 500, L17 Balland, C., Baumont, S., Basa, S., et al. 2009, A\&A, 507, 85 Benetti, S., Cappellaro, E., Mazzali, P. A., et al. 2005, ApJ, 623, 1011 Benetti, S., Meikle, P., Stehle, M., et al. 2004, MNRAS, 348, 261 Blondin, S., \& Berlind, P. 2008, CBET, 1424, 1

Blondin, S., Dessart, L., Leibundgut, B., et al. 2006, AJ, 131, 1648 Blondin, S., Kasen, D., Röpke, F. K., Kirshner, R. P., \& Mandel, K. S. 2011a, MNRAS, in press, doi:10.1111/j.1365-2966.2011.19345.x Blondin, S., Mandel, K. S., \& Kirshner, R. P. 2011b, A\&A, 526, A81 Blondin, S., \& Tonry, J. L. 2007, ApJ, 666, 1024

Branch, D. 1987, ApJ, 316, L81

Branch, D., Dang, L. C., \& Baron, E. 2009, PASP, 121, 238

Branch, D., Dang, L. C., Hall, N., et al. 2006, PASP, 118, 560

Branch, D., Drucker, W., \& Jeffery, D. J. 1988, ApJ, 330, L117

Branch, D., Garnavich, P., Matheson, T., et al. 2003, AJ, 126, 1489

Bronder, T. J., Hook, I. M., Astier, P., et al. 2008, A\&A, 477, 717 Chotard, N., Gangler, E., Aldering, G., et al. 2011, A\&A, 529, L4 Conley, A., Guy, J., Sullivan, M., et al. 2011, ApJS, 192, 1

Cristiani, S., Cappellaro, E., Turatto, M., et al. 1992, A\&A, 259, 63 Elias-Rosa, N., Benetti, S., Cappellaro, E., et al. 2006a, CBET, 608, 1 Elias-Rosa, N., Benetti, S., Cappellaro, E., et al. 2006b, MNRAS, 369, 1880 Fabricant, D., Cheimets, P., Caldwell, N., \& Geary, J. 1998, PASP, 110, 79 Filippenko, A. V., Foley, R. J., \& Desroches, L. 2003, IAU Circ., 8175, 2 Filippenko, A. V., Richmond, M. W., Branch, D., et al. 1992a, AJ, 104, 1543 Filippenko, A. V., Richmond, M. W., Matheson, T., et al. 1992b, ApJ, 384, L15 Folatelli, G. 2004, New Astron. Rev., 48, 623

Folatelli, G., Phillips, M. M., Burns, C. R., et al. 2010, AJ, 139, 120 Foley, R. J., Filippenko, A. V., Aguilera, C., et al. 2008a, ApJ, 684, 68 Foley, R. J., Filippenko, A. V., \& Jha, S. W. 2008b, ApJ, 686, 117 Foley, R. J., \& Kasen, D. 2011, ApJ, 729, 55

Foley, R. J., Matheson, T., Blondin, S., et al. 2009, AJ, 137, 3731

Foley, R. J., Narayan, G., Challis, P. J., et al. 2010, ApJ, 708, 1748

Foley, R. J., Perley, D., Bloom, J. S., \& Prochaska, J. X. 2005, IAU Circ., 8581, 3

Foley, R. J., Silverman, J. M., Moore, M., \& Filippenko, A. V. 2006, CBET, 604, 1

Ganeshalingam, M., Li, W., Filippenko, A. V., et al. 2010, ApJS, 190, 418 
Garavini, G., Aldering, G., Amadon, A., et al. 2005, AJ, 130, 2278

Garavini, G., Folatelli, G., Goobar, A., et al. 2004, AJ, 128, 387

Garavini, G., Folatelli, G., Nobili, S., et al. 2007a, A\&A, 470, 411

Garavini, G., Nobili, S., Taubenberger, S., et al. 2007b, A\&A, 471, 527

Garnavich, P. M., Bonanos, A. Z., Krisciunas, K., et al. 2004, ApJ, 613, 1120

Gómez, G., \& López, R. 1998, AJ, 115, 1096

Gurugubelli, U. K., Anupama, G. C., \& Sahu, D. K. 2006, CBET, 790, 1

Hachinger, S., Mazzali, P. A., \& Benetti, S. 2006, MNRAS, 370, 299

Hamuy, M., Maza, J., Pinto, P. A., et al. 2002, AJ, 124, 417

Hamuy, M., Phillips, M. M., Suntzeff, N. B., et al. 1996a, AJ, 112, 2408

Hamuy, M., Phillips, M. M., Suntzeff, N. B., et al. 1996b, AJ, 112, 2391

Harutyunyan, A., Benetti, S., Fiorenzano, A., \& Stanishev, V. 2008, CBET, 1438,1

Hicken, M., Challis, P., Jha, S., et al. 2009a, ApJ, 700, 331

Hicken, M., Wood-Vasey, W. M., Blondin, S., et al. 2009b, ApJ, 700, 1097

Höflich, P., Khokhlov, A., Wheeler, J. C., et al. 1996, ApJ, 472, L81

Hook, I. M., Howell, D. A., Aldering, G., et al. 2005, AJ, 130, 2788

Howell, D. A. 2001, ApJ, 554, L193

Jeffery, D. J., Leibundgut, B., Kirshner, R. P., et al. 1992, ApJ, 397, 304

Jha, S., Garnavich, P., Challis, P., et al. 1999a, IAU Circ., 7206, 1

Jha, S., Garnavich, P. M., Kirshner, R. P., et al. 1999b, ApJS, 125, 73

Jha, S., Kirshner, R. P., Challis, P., et al. 2006, AJ, 131, 527

Kasen, D., \& Plewa, T. 2007, ApJ, 662, 459

Kasen, D., \& Woosley, S. E. 2007, ApJ, 656, 661

Kasliwal, M. M., Ofek, E. O., Gal-Yam, A., et al. 2008, ApJ, 683, L29

Kelly, B. C. 2007, ApJ, 665, 1489

Kessler, R., Becker, A. C., Cinabro, D., et al. 2009, ApJS, 185, 32

Kirshner, R. P., Jeffery, D. J., Leibundgut, B., et al. 1993, ApJ, 415, 589

Konishi, K., Frieman, J. A., Goobar, A., et al. 2011, arXiv:1103.2497

Kotak, R., Meikle, W. P. S., Pignata, G., et al. 2005, A\&A, 436, 1021

Krisciunas, K., Garnavich, P. M., Stanishev, V., et al. 2007, AJ, 133, 58

Krisciunas, K., Li, W., Matheson, T., et al. 2011, AJ, 142, 74

Leibundgut, B., Kirshner, R. P., Filippenko, A. V., et al. 1991, ApJ, 371, L23

Leibundgut, B., Kirshner, R. P., Phillips, M. M., et al. 1993, AJ, 105, 301

Leloudas, G., Stritzinger, M. D., Sollerman, J., et al. 2009, A\&A, 505, 265

Leonard, D. C. 2005, CBET, 345, 1

Leonard, D. C., Li, W., Filippenko, A. V., Foley, R. J., \& Chornock, R. 2005, ApJ, 632,450

Li, W. D., Qiu, Y. L., Qiao, Q. Y., et al. 1999, AJ, 117, 2709

Maeda, K., Benetti, S., Stritzinger, M., et al. 2010, Nature, 466, 82

Maeda, K., Leloudas, G., Taubenberger, S., et al. 2011, MNRAS, 413, 3075

Mandel, K. S., Narayan, G., \& Kirshner, R. P. 2011, ApJ, 731, 120

Mandel, K. S., Wood-Vasey, W. M., Friedman, A. S., \& Kirshner, R. P. 2009, ApJ, 704, 629

Matheson, T., Kirshner, R. P., Challis, P., et al. 2008, AJ, 135, 1598

Maund, J. R., Höflich, P., Patat, F., et al. 2010, ApJ, 725, L167

Maza, J., Hamuy, M., Wischnjewsky, M., et al. 1992, IAU Circ., 5555, 1

Mazzali, P. A., Danziger, I. J., \& Turatto, M. 1995, A\&A, 297, 509

Mazzali, P. A., Lucy, L. B., Danziger, I. J., et al. 1993, A\&A, 269, 423

McNaught, R. H., Parker, Q. A., della Valle, M., et al. 1992, IAU Circ., 5569, 1

Nordin, J., Östman, L., Goobar, A., et al. 2011a, A\&A, 526, A119
Nordin, J., Östman, L., Goobar, A., et al. 2011b, ApJ, 734, 42

Nugent, P., Phillips, M., Baron, E., Branch, D., \& Hauschildt, P. 1995, ApJ, 455, L147

Nugent, P., \& Wang, L. 2001, IAU Circ., 7614, 3

Pastorello, A., Mazzali, P. A., Pignata, G., et al. 2007, MNRAS, 377, 1531

Patat, F., Benetti, S., Cappellaro, E., et al. 1996, MNRAS, 278, 111

Patat, F., Contreras, C., Prieto, J., et al. 2001, IAU Circ., 7680, 1

Peek, J. E. G., \& Graves, G. J. 2010, ApJ, 719, 415

Perlmutter, S., Mazzali, P. A., Pignata, G., et al. 1999, ApJ, 517, 565

Phillips, M. M. 1993, ApJ, 413, L105

Phillips, M. M., Lira, P., Suntzeff, N. B., et al. 1999, AJ, 118, 1766

Pignata, G., Benetti, S., Mazzali, P. A., et al. 2008, MNRAS, 388, 971

Pinto, P. A., \& Eastman, R. G. 2001, New Astron., 6, 307

Quimby, R., Castro, F., Edelmann, H., \& Riley, V. 2006a, CBET, 751, 1

Quimby, R., Höflich, P., Kannappan, S. J., et al. 2006b, ApJ, 636, 400

Rau, A., Kulkarni, S. R., Law, N. M., et al. 2009, PASP, 121, 1334

Reindl, B., Tammann, G. A., Sandage, A., \& Saha, A. 2005, ApJ, 624, 532

Riess, A. G., Filippenko, A. V., Challis, P., et al. 1998, AJ, 116, 1009

Riess, A. G., Kirshner, R. P., Schmidt, B. P., et al. 1999, AJ, 117, 707

Riess, A. G., Macri, L., Casertano, S., et al. 2011, ApJ, 730, 119

Riess, A. G., Press, W. H., \& Kirshner, R. P. 1996, ApJ, 473, 88

Riess, A. G., Strolger, L.-G., Casertano, S., et al. 2007, ApJ, 659, 98

Salvo, M. E., Benetti, S., Kjaergaard, P., \& Greve, T. R. 1999, IAU Circ., 7238,

Salvo, M. E., Cappellaro, E., Mazzali, P. A., et al. 2001, MNRAS, 321, 254

Sauer, D. N., Mazzali, P. A., Blondin, S., et al. 2008, MNRAS, 391, 1605

Schlegel, D. J., Finkbeiner, D. P., \& Davis, M. 1998, ApJ, 500, 525

Selj, J., Sharapov, D., Somero, A., et al. 2006, CBET, 570, 1

Silverman, J. M., Foley, R. J., \& Filippenko, A. V. 2007, CBET, 818, 3

Silverman, J. M., Wong, D., Filippenko, A. V., \& Chornock, R. 2006, CBET, 765,1

Stanishev, V., Goobar, A., Benetti, S., et al. 2007, A\&A, 469, 645

Stritzinger, M., Hamuy, M., Suntzeff, N. B., et al. 2002, AJ, 124, 2100

Sullivan, M., Guy, J., Conley, A., et al. 2011, ApJ, 737, 102

Suntzeff, N. B., \& Smith, R. C. 2000, IAU Circ., 7506, 2

Taubenberger, S., Hachinger, S., Pignata, G., et al. 2008, MNRAS, 385, 75

Tripp, R. 1998, A\&A, 331, 815

Turatto, M., Benetti, S., Cappellaro, E., et al. 1996, MNRAS, 283, 1

Turatto, M., Piemonte, A., Benetti, S., et al. 1998, AJ, 116, 2431

Umbriaco, G., Pietrogrande, T., di Mille, F., et al. 2007, CBET, 1174, 1

Valentini, G., Di, C. E., Massi, F., et al. 2003, ApJ, 595, 779

Walker, E. S., Hook, I. M., Sullivan, M., et al. 2011, MNRAS, 410, 1262

Wang, L. 2001, IAU Circ., 7640, 2

Wang, L., Baade, D., Höflich, P., et al. 2003, ApJ, 591, 1110

Wang, L., Baade, D., Höflich, P., et al. 2006, ApJ, 653, 490

Wang, L., \& Wheeler, J. C. 2008, ARA\&A, 46, 433

Wang, X., Filippenko, A. V., Ganeshalingam, M., et al. 2009a, ApJ, 699, L139

Wang, X., Li, W., Filippenko, A. V., et al. 2009b, ApJ, 697, 380

Wells, L. A., Phillips, M. M., Suntzeff, B., et al. 1994, AJ, 108, 2233

Wood-Vasey, W. M., Miknaitis, G., Stubbs, C. W., et al. 2007, ApJ, 666, 694

Zheng, C., Romani, R. W., Sako, M., et al. 2008, AJ, 135, 1766 